TITLE:

\title{
Molecular dynamics simulations of cluster impacts on solid targets: implantation, surface modification, and sputtering
}

$\operatorname{AUTHOR}(S):$

Aoki, Takaaki

\section{CITATION:}

Aoki, Takaaki. Molecular dynamics simulations of cluster impacts on solid targets:

implantation, surface modification, and sputtering. Journal of Computational Electronics 2013, 13(1): 108-121

ISSUE DATE:

2013-09-12

URL:

http://hdl.handle.net/2433/196774

\section{RIGHT:}

The final publication is available at Springer via http://dx.doi.org/10.1007/s10825-0130504-5; This is not the published version. Please cite only the published version.; この論文 は出版社版でありません。引用の際には出版社版をご確認ご利用ください。 


\title{
Molecular dynamics simulations of cluster impacts on solid targets: implantation, surface modification, and sputtering
}

Takaaki Aoki

\author{
Department of Electronic Science and Engineering, Graduate School of \\ Engineering, Kyoto University
}

$+81-75-383-7136$

$+81-75-383-7366$

aoki@sakura.nucleng.kyoto-u.ac.jp

\begin{abstract}
The collisions of cluster projectiles on solid targets were studied using molecular dynamics (MD) simulations. The penetration range and damage induced by small boron and carbon cluster implantations are compared with those induced by monomers with the same energy per atom. The simulations indicated enhanced penetration depth and the formation of dense track damage at the surface region. In addition, large argon and fluorine clusters (up to 1 million atoms) have shown effects such as crater formation and low-damage surface etching of silicon. The MD simulations revealed that cluster penetration and crater formation depends not on the total incident energy, but on the incident energy per atom, and that the damage threshold for the argon cluster is several eV/atom. In the impact of a very large and slow fluorine cluster on silicon, an enhancement of chemical reactivity at the near surface region was observed because of the high density of fluorine molecules depositing kinetic energy.
\end{abstract}

Keywords: cluster ion beam, molecular dynamics simulation, implantation, sputtering, surface modification

\section{Introduction}

Collisions of atomic or molecular ion clusters with solid targets are expected to result in different impact effects compared with those induced by monomer ions. In the late 1980s, the Yamada group developed two cluster ion beam systems for industrial applications $[1,2]$. One type was based on small molecules such as decaborane $\left(\mathrm{B}_{10} \mathrm{H}_{14}\right)$ and fullerene $\left(\mathrm{C}_{60}\right)$. $\mathrm{A} \mathrm{B}_{10} \mathrm{H}_{14}$ molecule accelerated to $5 \mathrm{keV}$ has a similar penetration depth to a single 500 $\mathrm{eV}$ boron atom, which has the same energy carried by each constituent boron atom in $\mathrm{B}_{10} \mathrm{H}_{14}$. This is the 'equivalent low energy' effect. However, it was found that the damage caused by cluster implantation differs from that caused by the monomer ion, which is advantageous for formation of shallow junctions in large-scale integrated circuits [3].

The second type of cluster ion beam is the 'gas cluster ion beam.' In this case, a high-pressure gas is injected into a vacuum system through a small nozzle. Because of adiabatic expansion in the nozzle, the atoms or molecules in the gas are cooled and coalesce to form clusters, which are ionized and accelerated into the target. The size of the gas cluster is much larger than the aforementioned small molecule cluster, ranging from several hundred particles to more than 100,000 [4]. These very large projectiles exhibit higher sputtering yields and increased surface smoothing compared with monomer impact. The mechanisms of cluster collisions on solid targets have been studied experimentally. For example, clusterbombarded surfaces revealed large damage tracks or characteristic crater shapes [5] that depend on cluster size and incident energy. However, these observations represent only the final product of the collisional process, and it is difficult to know the exact cluster size and energy. In contrast, computed molecular dynamics (MD) simulations of cluster impacts depict the entire collisional process with high spatial and temporal resolution and can establish a model that correlates with experimental results. In this article, MD simulations of cluster 
impacts on solid targets are discussed, including projectile penetration, damage generation, and sputtering of target materials for various constituents, cluster sizes, and energies.

\section{MD simulations of cluster impacts}

For single-particle or monomer ion collisions, computer simulations based on the binary collision approximation [6] have successfully described the generation of knocked-on atoms and the development of collision cascades. It has allowed us to understand implantation, damage formation, and mixing and sputtering, for both single and complex materials that are more difficult to solve analytically. On the other hand, modeling cluster impacts requires a large number of interactions that occur in a narrow time and space domain, not only between the cluster and target atoms, but also for the atoms inside the cluster and the target near the impact point. Thus, the motion of all atoms around the impact point should be taken into consideration. One should calculate the interactions among all atoms in the system, and numerically solve Newton's equations of motion to trace the time development of the atomic positions and velocities. This is essentially MD, and this why MD simulation is appropriate for the analysis of cluster impact.

In this article, several variations of MD simulations are used to characterize cluster impacts on solid targets. Details of the MD simulations have been reported elsewhere $[7,8]$. The interatomic potentials adopted for the simulations are summarized in Table 1. It should be noted that, in order to treat high-energy particle collisions, the potential energy at short atomic distances should be large and become infinite as the distance approaches zero. Thus, the Ziegler-Biersack-Littmark (ZBL) screening function should be used instead of the Tersoff potential for carbon-carbon interactions. Additionally, argon interactions are described solely with the ZBL repulsive potential because the binding energy of argon and other atoms discussed here are negligible compared with the incident energy of the particles and binding energy of the target materials. With adequate computational time and resources, the number of atoms in the targets ranged from $10^{4}$ to $10^{8}$, depending on the cluster size and energy of the projectile. To absorb the excess energy of the cluster impact, a thermal bath region governed by Langevin dynamics surrounds the target bottom (and the side edges, if necessary).

\section{Results and discussion}

\section{Range and damage by cluster implantation}

Fig. 1 shows snapshots of carbon monomers and fullerene impacting diamond (100) substrates [14]. In Fig. 1, each carbon atom is accelerated with 20, 200, or $2000 \mathrm{eV} /$ atom, so for an 'equivalent low energy' the fullerenes impact the targets with $1.2,12$, and $120 \mathrm{keV}$ of total kinetic energy, respectively. The trajectories and final positions of the projectile atoms are indicated as small black dots and open circles, and displaced target carbon atoms are indicated in gray. The upper pictures of Fig. 1 were made by overlapping 60 individual snapshots of carbon monomer impacts, whereas the lower pictures represent the trajectories of the 60 atoms in a single fullerene impact. Additionally, the mean penetration depth $\left(R_{\mathrm{p}}\right)$ of projectile carbon atoms implanted as monomer or fullerene are shown in Fig. 2. As shown in Figs. 1 and 2, when the incident energy per atom is higher than $1 \mathrm{keV}$, the same $R_{\mathrm{p}}$ is obtained by both monomer and fullerene collisions. However, when the incident energy per atom is less than $1 \mathrm{keV}$, a larger $R_{\mathrm{p}}$ is observed for fullerene impacts. It is possible that for monomer carbon impacts in this energy range, some projectiles could be reflected at the target surface. However, when 60 carbon atoms impact the target at once as a cluster, multiple collisions will occur without reflection, which results in an enhancement of $R_{\mathrm{p}}$.

Two mechanisms are assumed for the enhancement of $R_{\mathrm{p}}$ by multiple collisions. The first one is the acceleration effect, where the first contacting projectile atom penetrates into the target by receiving kinetic energy under multiple collisions from the cluster atoms that follow it. The second mechanism is the clearing-way effect, where the first contacting atom penetrates the target surface and the target atom is knocked on; then the remaining cluster atoms penetrate deeper into the target without collision and energy loss. In this energy region, the incident cluster spreads isotropically from the impact point, so that a spherical crater or damaged region is created. When the incident energy is as low as $20 \mathrm{eV} /$ atom, a fullerene 
cannot penetrate the diamond target, even with the help of multiple collisions. In this case, the incident fullerene first deforms and then bounces from or sticks to the target.

There is one model that helps us to understand the critical energy per atom where $R_{\mathrm{p}}$ of the monomers and the cluster diverge. In Fig. 3, there are profiles of shadow cones formed by carbon atoms impacting on a diamond substrate with various incident energies. The cones are drawn using the same interatomic potential as for the MD simulations in Figs. 1 and 2. Each line in Fig. 3 indicates the trajectory of an incident carbon atom, which collides with the target carbon atoms at $(0,0)$ with various impact parameters. The black circles are the coordinates of the target atoms in the diamond structure, which indicate how far the incident carbon can penetrate into the substrate until the next collision with a substrate atom. In cases of low-energy ion impacts such as at 100 and $500 \mathrm{eV}$, the incident atom is largely scattered at the first collision and then is expected to collide with the next substrate atom, which resides shallower than the depth of a one unit cell. If a cluster impacts on the surface with such low incident energy, the first atom to collide with a surface atom is largely scattered in a lateral direction and the atom can reside in a shallow region, which then causes a lateral dispersion of the cluster and a large number of collisions between the first incident atom and the ones that follow it. As the incident energy increases, the cross-section of the impact decreases and the incident atom penetrates into the deep region of the substrate without secondary collision with target atoms.

As shown in Figs. 1 and 2, the differing $R_{\mathrm{p}}$ between the monomer and the fullerene occurs for energies less than several $\mathrm{keV} / \mathrm{atom}$. However, the non-linearity in damage formation occurs at higher energy. Fig. 4 shows the depth profiles of displacements caused by carbon monomer and fullerene $0.1 \mathrm{ps}$ after impact at incident energies of $200 \mathrm{eV} /$ atom and 2 $\mathrm{keV} / \mathrm{atom}$. The number of displacements caused by one carbon projectile is used to assess damage; the damage profile by fullerene impact is calculated by dividing the total number of displacements by 60. Fig. 4 indicates that when the incident energy is $200 \mathrm{eV} / \mathrm{atom}$, a fullerene can penetrate into the target deeper than the monomer and causes a larger number of displacements surrounding the incident impact, as shown in Fig. 1. When the incident energy is $2 \mathrm{keV} /$ atom, the carbon monomers and the fullerenes have the same $R_{\mathrm{p}}$, but the damage depth profiles differ. The right plot in Fig. 4 shows that a fullerene causes high-density damages at a shallow region, but also causes interspersed point defects at the bottom of the target in a manner similar to carbon monomer impacts. The cascade-overlapping model [15] could be used to explain the non-linearity in damage formation. In the case of high-energy fullerene impact, each projectile is assumed to penetrate the target individually, generating knocked-on displacements along its trajectory. In monomer impacts, some of the knocked-on target atoms return to their original lattice sites. However, when many collision cascades are caused simultaneously in a narrow area by cluster impact, most of the knocked-on atoms cannot return to their original lattice sites because the surrounding atoms are also displaced. As the incident atoms of fullerenes penetrate into deep target regions, the spatial coherency of the cascade diminishes and interspersed defects are created.

Similar interpretations could be applied to polyatomic boron cluster implantation using decaborane $\left(\mathrm{B}_{10} \mathrm{H}_{14}\right)$ and octadecaborane $\left(\mathrm{B}_{18} \mathrm{H}_{22}\right)$. Fig. 5 plots the incident energy dependence of the degree of enhancement of $R_{\mathrm{p}}$ for various sizes of boron clusters implanted into silicon [16]. At low implant energies, there are significant differences in $R_{\mathrm{p}}$ between the monomers and the clusters. The threshold energy for $R_{\mathrm{p}}$ nonlinearity is $500 \mathrm{eV} /$ atom, which is less than that for the carbon clusters on diamond. These results suggest that the nonlinearity of cluster ion implantation depends on cluster size, energy, and the combination of projectile and target materials.

MD simulation provides the precise coordinates of all atoms in the system, which allows us to analyze local structures around specific atoms such as the folding number, interstitials, and vacancies. Fig. 6 shows depth distributions of vacancies and interstitials formed by $\mathrm{B}_{1}$ and $\mathrm{B}_{10}$ implantation with an incident energy of $200 \mathrm{eV} /$ atom. The definitions of vacancy and interstitial are taken from Peréz-Martín et al. [17]. For $\mathrm{B}_{10}$, the profiles have been normalized to indicate the number of vacancies or interstitials created by one $\mathrm{B}$ atom in a cluster. The maximum depth ranges for vacancies and interstitials formed by $\mathrm{B}_{1}$ and $\mathrm{B}_{10}$ impacts are similar. However, $\mathrm{B}_{10}$ impact causes a very high density of defects from the surface to a depth of $2 \mathrm{~nm}$. This distribution is comparable to the mean implant depth of B monomers and clusters. Considering the density of interstitials shown in Fig. 5, $\mathrm{B}_{10}$ impact creates about 15 interstitials per $0.2 \mathrm{~nm}$ in depth. From these results, it is expected that the 
impact point is made completely amorphous by only one $\mathrm{B}_{10}$ ion collision.

Fig. 7 plots radial distributions of substrate $\mathrm{Si}$ atoms around vacancies and interstitials. In the case of $\mathrm{B}_{1}$ impact, the distribution around vacancies shows distinct peaks and valleys. Compared with the radial distribution function of the bulk Si crystal, each peak in the $\mathrm{B}_{1}$ distribution shifts to shorter distances, but shows good correlation with the one in the $\mathrm{Si}$ crystal. As for the distribution around interstitials at $\mathrm{B}_{1}$ impact, the second peak is observed at $0.31 \mathrm{~nm}$ with a similar intensity to the first peak. Those two distributions indicate that, at the impact of $\mathrm{B}_{1}$, only one knocked-on atom is displaced from its lattice site while the surrounding atoms maintain lattice positions. This results in the formation of point defects having certain structures [17]. Following the impact of $B_{10}$, the peak-and-valley structure is not found around vacancies within $0.3 \mathrm{~nm}$. This result means that the lattice structures before and after impact have no correlation; that is, the damaged structure is fully amorphous. The distribution around interstitials also becomes broad as the distance increases. From these results, it can be concluded that the implant region is made fully amorphous by only one $\mathrm{B}_{10}$ impact at $200 \mathrm{eV} /$ atom. As reported previously [18], the creation of an amorphous surface region with sufficient depth relative to the boron implant range can reduce transient-enhanced diffusion. The "self-amorphous" damage created by $\mathrm{B}_{10}\left(\right.$ and $\left.\mathrm{B}_{18}\right)$ is known to enhance the formation of high-performance shallow junctions $[19,20]$.

\section{Large gas cluster impact and crater formation}

Gas cluster ion beam techniques provide high-intensity and large-size cluster ions. As mentioned above, they are generated by high-pressure nozzles where adiabatic expansion causes the gas to condense into large clusters ranging from several hundred up to a million atoms. Fig. 8 shows snapshots of an $\mathrm{Ar}_{2000}$ cluster impacting on a $\mathrm{Si}(100)$ solid surface with a total energy of $20 \mathrm{keV}$ (so each constituent atom carries only $10 \mathrm{eV}$ ). Even at this low energyper-atom, the incident cluster penetrates the target intact as a cluster because of the proximity effect. The cluster atom that first contacts the target can knock-on the surface target atom and be implanted into the target. The remaining cluster atoms that follow can then penetrate deeper without energy loss, which enhances the penetration depth of the cluster. The implanted cluster atoms spread under the target surface. A large number of target surface atoms are displaced hemispherically from the impact area because of the multiple collisions. Almost all of the Ar atoms in the incident cluster leave the target and the crater-like damage is left on the surface. This characteristic crater formation has been confirmed by MD simulations of other materials [21-23] and by experiments [24, 25]. During the crater formation process, some atoms at the rim may gain enough energy to leave the target, which is a feature of sputtering with huge clusters [26-28].

Figs. 9 and 10 demonstrate impacts on irregular surface structures [29]. The initial surface has a one-dimensional sinusoidal structure with a 4-nm peak-to-valley height and an 8-nm wavelength. A $20 \mathrm{keV} \mathrm{Ar} 2000$ cluster impacts along the surface normal and at the top of the crest. With only a single impact, the crest is completely removed, flattening the surface profile. The atoms residing in the crest filled the valleys on both sides. These results indicate that a large cluster impact has the capability to move many surface atoms simultaneously, and the displaced surface atoms smooth out the target surface. This is essentially the mechanism of surface smoothing with cluster bombardment, which has been demonstrated by experiments [30-32] as well as simulations [33].

Fig. 11 plots the surface profiles, and the depth and opening radii of craters caused by $\mathrm{Ar}_{2000}$ clusters with various incident energies. For total incident energies higher than $10 \mathrm{keV}$, the crater depth and radius increase proportionally with the cubic root of the incident energy. In this range, almost all of the incident kinetic energy is consumed in crater formation, and thus the crater shape keeps its aspect ratio. In contrast, the crater depth rapidly decreases for energies less than $10 \mathrm{keV}$. At $10 \mathrm{keV}$, each Ar atom in the cluster carries only $5 \mathrm{eV}$, which is similar to the displacement energy for the Si target atoms. In this energy regime, the firstcontacting Ar atom cannot penetrate the surface but is reflected instead; thus it contributes to the disruption of the cluster on the target surface. Therefore, the threshold condition for crater formation is determined by the incident energy-per-atom rather than the total energy of the cluster [34].

Fig. 12 shows snapshots of various sizes of argon gas clusters impacting on silicon. Each cluster has the same total incident energy of $20 \mathrm{keV}$, but the surface damage depends on 
cluster size. When a cluster is relatively small, it penetrates the target surface and causes multiple collisions, similar to Fig. 1. The difference in the shape of the crater is significant when comparing $\operatorname{Ar}_{20}(1 \mathrm{keV} /$ atom $)$ and $\mathrm{Ar}_{200}(100 \mathrm{eV} /$ atom $)$, but not when comparing $\mathrm{Ar}_{200}$ and $\mathrm{Ar}_{2000}(10 \mathrm{eV} /$ atom). Fig. 13 shows surface profiles and crater depths caused by $\mathrm{Ar}$ clusters of various sizes that all have a total incident energy of $20 \mathrm{keV}$. There is a specific cluster size around 5000 where the crater depth rapidly decreases. In this case, the incident energy per atom is $4 \mathrm{eV}$, which supports the above discussion about a threshold condition for crater generation. On the other hand, the change of crater depth is relatively small for cluster sizes ranging from 20 to 2000 . In this size regime, a cluster could be treated as a continuous material that transfers its kinetic energy to surrounding target atoms isotropically. The cluster size effect on crater formation has been compared with experimental results. Scanning tunneling microscope images indicate that surfaces bombarded with different sizes of clusters had a threshold energy-per-atom or size for crater formation [35]. Other experiments have demonstrated that the roughness of the surface can be controlled by changing the cluster size [36-38].

Fig. 14 shows the surface profiles and crater depths caused by $20 \mathrm{keV} \mathrm{Ar}_{2000}$ impacts at various incident angles [39]. As the incident angle increases, the crater shape becomes asymmetric and shallower. When the incident angle is more than 70 degrees from the surface normal, the crater is no longer formed. The conditions for crater formation thus can be explained by considering the normal component of incident energy-per-atom, which is calculated as 5, 2.5, 1.2 or $0.3 \mathrm{eV} /$ atom for $45,60,70$ or 80 degrees, respectively. These MD simulations suggest that the energy threshold to cause surface damage is mainly dominated by the incident energy per atom rather than total energy. When the incident energy-per-atom is as low as several $\mathrm{eV}$ with respect to the surface normal, each constituent cluster atom does not penetrate the target surface even with the help of high-density multiple collision effects. The glancing angle bombardment by large clusters also smooths the surface. When a target with a small block attached to the surface is bombarded with a large cluster at a large incident angle that is nearly in the surface plane, the target surface plane is not damaged because of the aforementioned reasons, but the kinetic energy of the cluster is transferred entirely to the block, which will be removed $[39,40]$. This effect has been used to polish the sidewalls of etched structures that are oriented along the surface normal [41].

The fundamental mechanisms of multiple collisions at a low incident energy-per-atom regime were studied by the impacts of simulated clusters. Fig. 15 shows snapshots of $20 \mathrm{keV}$ $\operatorname{Ar}_{2000}(10 \mathrm{eV} /$ atom) clusters with various shapes and densities impacting on Si (100) target surfaces [42]. The particle density was varied by a factor of 4 in both the $x y$ - and $z$-axes, independently. Thus, Fig. 15(b) corresponds to a factor of 16 lower areal particle density relative to Fig. 15(a), and the volume density in Fig. 15(d) is a factor of 64 less than in 15(a). In another perspective, the $x y$ and $z$ scaling corresponds to changes in spatial and temporal coherence, respectively. The crater depth dependence on $x y$ and $z$ scaling factors is shown in Fig. 16. Figs. 15 and 16 indicate that the penetration depth and crater formation effects are much reduced when the cluster expands in the lateral direction rather than along the normal direction. The energy densities at the 1.26, 1.5, 2.0, and 4.0 xy scaling factors are roughly calculated as $6.3,4.4,2.5$, and $0.625 \mathrm{eV} /$ atom/area, respectively, which agrees with the results discussed in Figs. 11, 13, and 14.

\section{Chemical etching by reactive cluster impact}

Multiple collision and high-density atomic bombardment could enhance chemical reactivity of surface modification processes. Fig. 17 shows snapshots of neon and fluorine clusters impacting a silicon target [43]. The total incident energy is kept at $6 \mathrm{keV}$, while the cluster size varies from 60 to 6000 . We see that both neon and fluorine clusters cause craterlike damage when the cluster size is 60 or 600 and the incident energy is at least 100 or 10 eV/atom, respectively. When the cluster size is very large and the incident energy is very low, such as for $\mathrm{Ne}_{6000}$ or $\left(\mathrm{F}_{2}\right)_{3000}$ with $1 \mathrm{eV} /$ atom, the incident cluster does not produce craters on the target, but creates only a small dimple. Additionally, the snapshots of fluorine cluster impacts indicate that the fluorine molecules in the incident cluster dissociate during the collision, forming silicon-fluoride precursors at the impact point.

The number of sputtered $\mathrm{Si}$ atoms and the distribution of silicon-fluoride composites are tabulated in Fig. 18 for 100 trials that were carried out for each impact 
condition. The average values of each sputtered species are then calculated. The sputtering process could be controlled by changing the cluster size and the incident energy per atom, while keeping the total incident energy constant. In the case of small and fast clusters such as $\left(\mathrm{F}_{2}\right)_{30}$ with $100 \mathrm{eV} /$ atom, silicon monomers, dimers, and larger silicon clusters are the major sputtered components instead of silicon fluorides. These results are similar to those obtained from sputtering with $\mathrm{Ne}_{60}$ at $100 \mathrm{eV} /$ atom. This suggests that momentum transfer by direct collisions dominates the mechanism of sputtering, even for $\left(\mathrm{F}_{2}\right)_{30}$ cluster impact. As the cluster size increases and the incident energy per atom decreases with a constant total kinetic energy, the chemical reactivity of the cluster and the target materials becomes significant. In these simulations, no Si sputtering was observed for $\mathrm{Ne}_{600}$ with $10 \mathrm{eV} /$ atom nor for $\mathrm{Ne}_{6000}$ with 1eV/atom; however, large fluorine clusters produced high Si sputtering yields. The major sputtered product shifts from silicon to silicon fluoride, which suggests that a chemical reaction is a key process of surface etching for the slow impacts of large reactive clusters. These clusters can provide a large amount of etchant material at high density over a short time. Subsequently, the clusters reside at a very shallow area of the target surface, where multiple collisions between the cluster and the target contribute to the formation and desorption of the etching product.

With recent improvements in computational resources, simulated impacts for very large cluster sizes and higher energy systems have been made possible. Fig. 19 shows the impact of a fluorine cluster with 1 million $\left(\left(\mathrm{F}_{2}\right)_{500 \mathrm{k}}\right)$ atoms impacting on a $\mathrm{Si}(100)$ target consisting of more than 130 million atoms [44]. Even at such large cluster impacts, similar collision processes to $\left(\mathrm{F}_{2}\right)_{3000}$ were observed, with a silicon sputtering yield of more than 12,000. However, Fig. 19 shows dislocations (indicated as blue spheres) that penetrate into the target and remain at a depth of more than $10 \mathrm{~nm}$ from the surface $77 \mathrm{fs}$ after impact. This was not observed with the smaller fluorine cluster impacts, such as $\left(\mathrm{F}_{2}\right)_{5000}$ and $\left(\mathrm{F}_{2}\right)_{50000}$ with $1 \mathrm{eV} /$ atom [45]. This may be due to generation of a highly compressed region surrounding the crater at the initial stage of cluster impact, which depends on total energy and mass of the projectile. The results imply that in order to achieve enhanced surface modification, surface sputtering and damage formation should be precisely controlled.

\section{Summary}

In this article, we have discussed various MD simulations of cluster impacts on solid targets. The results show that the species and total incident energy are key parameters for monomer ion beams, whereas cluster size and incident energy per atom are also significant collisional parameters for energetic clusters.

Fig. 20 summarizes the constituent, size, and energy-per-atom for clusters discussed in this article. For small and swift cluster bombardments, the interaction among cluster atoms is negligible and each projectile atom penetrates into the target in a manner similar to the individual monomer ions. Thus, we see that the penetration depth is almost the same as that for the monomer ion, and interspersed point defects due to collision cascades can be found at the end of range. However, cluster impacts generate a large number of secondary and tertiary knocked-on atoms in a narrow region simultaneously. These high-density cascades overlap with each other, resulting in dense damaged tracks around the impact point.

As the cluster sizes increases and the incident energy per atom decreases, collisions inside the cluster become significant. The cluster penetrates the target surface and stays intact, while the target atoms are compressed and are pushed away in an isotropically hemispherical direction through the multiple collisions among cluster and surface atoms. This collisional process leads to crater formation on a flat target surface, and it also smooths non-planar surfaces.

In this study, two types of energy per atom are discussed: one is where each cluster atom is regarded as an independent projectile, and the other is where a cluster atom cannot penetrate the target surface even with the help of the multiple collision effect. The latter energy seems to be related to the surface binding energy of the target. If the incident energy per atom is as low as this threshold energy, the cluster does not penetrate the target surface; however, it deposits some high-density particles and kinetic energy through the interface between cluster and target. From the viewpoint of physical interaction, this collisional process causes no damage. However, it contributes to the enhancement of chemical interactions, such 
as decomposition, adsorption, and desorption of reaction products.

The interactions between clusters and solid targets have been studied in various groups by means of both experiments and simulations, from fundamental aspects to industrial applications. The number of scientific and engineering fields where the cluster beam technique is expected to contribute is expanding. As for cluster projectiles, not only small molecule and gas clusters are being used, but also the generation and collision effects of large droplets formed by electrospray methods are being examined [46-48]. These droplets usually consist of a larger number of atoms/molecules compared with those in this article, and the collision effects are not clear. Conversely, cluster ion beam bombardment has been applied to organic materials for depth profiling [49]. This requires optimization of the interactions with organic materials in order to control damage mechanisms, chemical reactions, and etching with energetic cluster impact. Collisional effects for single impacts, as well as for accumulating effects realized by iterative impact simulation on the same target, are interesting topics for more precise comparison with experimental results. In addition, new multi-scale modeling techniques, such as combining MD and Monte-Carlo simulations for defect migration and annihilation, are expected to be introduced for further theoretical studies of cluster and surface interactions.

\section{References}

1. Yamada, I.: Progress, demands and prospects for advanced ion beam processing. Materials Chemistry and Physics. 54, 5-14 (1998).

2. Yamada, I., Khoury, J.: Cluster Ion Beam Processing: Review of Current and Prospective Applications. MRS Proceedings. 1354, mrss11-1354-ii03-01 (2011).

3. Goto, K.-I., Matsuo, J., Tada, Y., Tanaka, T., Momiyama, Y., Sugii, T., Yamada, I.: A high performance $50 \mathrm{~nm}$ PMOSFET using decaborane $\left(\mathrm{B}_{10} \mathrm{H}_{14}\right)$ ion implantation and 2 -step activation annealing process. International Electron Devices Meeting. IEDM Technical Digest. 00, 471-474 (1997).

4. Seki, T., Matsuo, J., Takaoka, G., Yamada, I.: Generation of the large current cluster ion beam. Nuclear Instruments and Methods in Physics Research Section B: Beam Interactions with Materials and Atoms. 206, 902-906 (2003).

5. Seki, T., Aoki, T., Tanomura, M., Matsuo, J., Yamada, I.: Energy dependence of a single trace created by $\mathrm{C}_{60}$ ion impact. Materials Chemistry and Physics. 54, 143-146 (1998).

6. Ziegler, J.F.: SRIM - The Stopping and Range of Ions in Matter, http://www.srim.org/. 7. Aoki, T.: Molecular Dynamics Simulation of Cluster Ion Impact on Solid Surface, http://hdl.handle.net/2433/8942, (2000).

8. Aoki, T., Seki, T., Matsuo, J.: Molecular dynamics simulations for gas cluster ion beam processes. Vacuum. 84, 994-998 (2010).

9. Tersoff, J.: New empirical approach for the structure and energy of covalent systems. Physical Review B. 37, 6991-7000 (1988).

10. Ziegler, J.F.: The Stopping and Range of Ions in Solids. Pergamon Pr (1985).

11. Stillinger, F., Weber, T.: Computer simulation of local order in condensed phases of silicon. Physical Review B. 31, 5262-5271 (1985).

12. Stillinger, F., Weber, T.: Fluorination of the dimerized $\mathrm{Si}(100)$ surface studied by molecular-dynamics simulation. Physical Review Letters. 62, 2144-2147 (1989).

13. Weakliem, P., Wu, C., Carter, E.: First-Principles-Derived Dynamics of a Surface Reaction: Fluorine Etching of Si(100). Physical Review Letters. 69, 200-203 (1992).

14. Aoki, T., Seki, T., Matsuo, J., Insepov, Z., Yamada, I.: Molecular dynamics simulation of a carbon cluster ion impacting on a carbon surface. Materials Chemistry and Physics. 54, 139-142 (1998).

15. Seki, T., Tanomura, M., Aoki, T., Matsuo, J., Yamada, I.: Size Dependence of Bombardment Characteristics Produced by Cluster Ion Beams. MRS Proceedings. 504, 93-98 (1998).

16. Aoki, T., Seki, T., Matsuo, J.: MD study of damage structures with poly-atomic boron cluster implantation. Extended Abstracts of the 7th International Workshop on Junction Technology. pp. 23-24. IEEE (2007).

17. Peréz-Martín, A.M.C., Domínguez-Vázquez, J., Jiménez-Rodríguez, J.J.: A MD study of low energy boron bombardment on silicon. Nuclear Instruments and 
Methods in Physics Research Section B: Beam Interactions with Materials and Atoms. 164-165, 431-440 (2000).

18. Kusaba, T., Shimada, N., Aoki, T., Matsuo, J., Yamada, I., Goto, K., Sugii, T.: Boron diffusion in ultra low-energy $\left(<1\right.$ keV/atom) decaborane $\left(\mathrm{B}_{10} \mathrm{H}_{14}\right)$ ion implantation. 1998 International Conference on Ion Implantation Technology. Proceedings. pp. 1258-1261. IEEE (1999).

19. Hamamoto, N., Umisedo, S., Nagayama, T., Tanjyo, M., Sakai, S., Nagai, N., Aoyama, T., Nara, Y.: Decaborane implantation with the medium current implanter. Nuclear Instruments and Methods in Physics Research Section B: Beam Interactions with Materials and Atoms. 237, 443-448 (2005).

20. Tanjyo, M., Nagayama, T., Onoda, H., Hamamoto, N., Umisedo, S., Koga, Y., Une, H., Maehara, N., Kawamura, Y., Hashino, Y., Nakashima, Y., Hashimoto, M., Tokoro, N., Nagai, N., Sekar, K., Krull, W.: Cluster Ion Implantation for Process Application Carbon Cluster co-Implantation-. AIP Conference Proceedings. 1321, 105-108 (2011).

21. Yamaguchi, Y., Gspann, J.: MD study on high-energy reactive carbon and oxygen cluster impact leading to surface erosion on diamond. Nuclear Instruments and Methods in Physics Research Section B: Beam Interactions with Materials and Atoms. 228, 309-314 (2005).

22. Rzeznik, L., Czerwinski, B., Garrison, B.J., Winograd, N., Postawa, Z.: Molecular dynamics simulations of sputtering of organic overlayers by slow, large clusters. Applied Surface Science. 255, 841-843 (2008).

23. Anders, C., Ziegenhain, G., Zimmermann, S., Urbassek, H.M.: Cluster-induced crater formation. Nuclear Instruments and Methods in Physics Research Section B: Beam Interactions with Materials and Atoms. 267, 3122-3125 (2009).

24. Matsuo, J., Seki, T., Aoki, T., Yamada, I.: Atomistic study of cluster collision on solid surfaces. Nuclear Instruments and Methods in Physics Research, Section B: Beam Interactions with Materials and Atoms. 206, 838-841 (2003).

25. Insepov, Z., Allen, L.P., Santeufemio, C., Jones, K., Yamada, I.: Crater formation and sputtering by cluster impacts. Nuclear Instruments and Methods in Physics Research Section B: Beam Interactions with Materials and Atoms. 206, 846-850 (2003).

26. Anders, C., Urbassek, H.M.: Cluster-size dependence of ranges of 100eV/atom Aun clusters. Nuclear Instruments and Methods in Physics Research Section B: Beam Interactions with Materials and Atoms. 228, 57-63 (2005).

27. Aoki, T., Seki, T., Ninomiya, S., Matsuo, J.: MD simulation study of the sputtering process by high-energy gas cluster impact. Applied Surface Science. 255, 944-947 (2008).

28. Aoki, T., Seki, T., Ninomiya, S., Ichiki, K., Matsuo, J.: Study of crater formation and sputtering process with large gas cluster impact by molecular dynamics simulations. Nuclear Instruments and Methods in Physics Research Section B: Beam 29. Interactions with Materials and Atoms. 267, 1424-1427 (2009).

Aoki, T., Matsuo, J.: Surface structure dependence of impact processes of gas cluster ions. Nuclear Instruments and Methods in Physics Research Section B: Beam Interactions with Materials and Atoms. 216, 185-190 (2004).

30. Kitani, H., Toyoda, N., Matsuo, J., Yamada, I.: Incident angle dependence of the sputtering effect of Ar-cluster-ion bombardment. Nuclear Instruments and Methods in Physics Research Section B: Beam Interactions with Materials and Atoms. 121, 489-492 (1997).

31. Toyoda, N.: Surface treatment of diamond films with Ar and O2 cluster ion beams. Nuclear Instruments and Methods in Physics Research Section B: Beam Interactions with Materials and Atoms. 148, 639-644 (1999).

32. Chen, H., Liu, S., Wang, X., Iliev, M., Chen, C., Yu, X., Liu, J., Ma, K., Chu, W. Smoothing of $\mathrm{ZnO}$ films by gas cluster ion beam. Nuclear Instruments and Methods in Physics Research Section B: Beam Interactions with Materials and Atoms. 241, 630-635 (2005).

33. Aoki, T., Matsuo, J.: Molecular dynamics simulations of sequential cluster ion impacts. Nuclear Instruments and Methods in Physics Research Section B: Beam Interactions with Materials and Atoms. 228, 46-50 (2005).

34. Aoki, T., Matsuo, J.: Molecular dynamics simulations of surface modification and 
damage formation by gas cluster ion impacts. Nuclear Instruments and Methods in Physics Research Section B: Beam Interactions with Materials and Atoms. 242, 517519 (2006).

35. Houzumi, S., Mochiji, K., Toyoda, N., Yamada, I.: Scanning Tunneling Microscopy Observation of Graphite Surfaces Irradiated with Size-Selected Ar Cluster Ion Beams. Japanese Journal of Applied Physics. 44, 6252-6254 (2005).

36. Nakamura, K., Houzumi, S., Toyoda, N., Mochiji, K., Mitamura, T., Yamada, I.: Cluster size dependences of bombardment effects using mass-selected gas cluster ion beams. Nuclear Instruments and Methods in Physics Research Section B: Beam Interactions with Materials and Atoms. 261, 660-663 (2007).

37. Ichiki, K., Ninomiya, S., Seki, T., Aoki, T., Matsuo, J.: The effects of cluster size on sputtering and surface smoothing of PMMA with gas cluster ion beams. Transactions of the Materials Research Society of Japan. 36, 309-312 (2011).

38. Toyoda, N., Yamada, I.: Reduction of irradiation damage using size-controlled nitrogen gas cluster ion beams. Nuclear Instruments and Methods in Physics Research Section B: Beam Interactions with Materials and Atoms. 273, 11-14 (2012). 39. Aoki, T., Matsuo, J.: Molecular dynamics study of glancing angle gas cluster irradiation on irregular-structured surfaces. Nuclear Instruments and Methods in Physics Research Section B: Beam Interactions with Materials and Atoms. 261, 639642 (2007).

40. Aoki, T., Matsuo, J.: Molecular dynamics simulations of surface smoothing and sputtering process with glancing-angle gas cluster ion beams. Nuclear Instruments and Methods in Physics Research Section B: Beam Interactions with Materials and Atoms. 257, 645-648 (2007).

41. Bourelle, E., Suzuki, A., Sato, A., Seki, T., Matsuo, J.: Sidewall polishing with a gas cluster ion beam for photonic device applications. Nuclear Instruments and Methods in Physics Research Section B: Beam Interactions with Materials and Atoms. 241, 622-625 (2005).

42. Aoki, T., Seki, T., Matsuo, J.: Study of density effect of large gas cluster impact by molecular dynamics simulations. Nuclear Instruments and Methods in Physics Research Section B: Beam Interactions with Materials and Atoms. 267, 2999-3001 (2009).

43. Aoki, T., Matsuo, J., Yamada, I.: Cluster size effect on reactive sputtering by fluorine cluster impact using molecular dynamics simulation. Nuclear Instruments and Methods in Physics Research Section B: Beam Interactions with Materials and Atoms. 180, 164-170 (2001).

44. Aoki, T., Seki, T., Matsuo, J.: Molecular dynamics simulation study of damage formation and sputtering with huge fluorine cluster impact on silicon. Nuclear Instruments and Methods in Physics Research Section B: Beam Interactions with Materials and Atoms. 303, 170-173 (2013).

45. Aoki, T., Seki, T., Matsuo, J.: Molecular dynamics simulations of large fluorine cluster impact on silicon with supersonic velocity. Nuclear Instruments and Methods in Physics Research Section B: Beam Interactions with Materials and Atoms. 269, 1582-1585 (2011).

46. Gamero-Castaño, M.: Retarding potential and induction charge detectors in tandem for measuring the charge and mass of nanodroplets. The Review of scientific instruments. 80, 053301 (2009).

47. Gamero-Castaño, M., Mahadevan, M.: Sputtering of silicon by a beamlet of electrosprayed nanodroplets. Applied Surface Science. 255, 8556-8561 (2009).

48. Gamero-Castaño, M., Mahadevan, M.: Sputtering yields of Si, SiC, and $\mathrm{B}_{4} \mathrm{C}$ under nanodroplet bombardment at normal incidence. Journal of Applied Physics. 106, 054305 (2009).

49. Ninomiya, S., Ichiki, K., Yamada, H., Nakata, Y., Seki, T., Aoki, T., Matsuo, J.: Analysis of organic semiconductor multilayers with Ar cluster secondary ion mass spectrometry. Surface and Interface Analysis. 43, 95-98 (2011).

\section{Tables}

Table 1. Interatomic potentials adopted in this paper 


\begin{tabular}{|l|l|}
\hline Atomic Pairs & Models \\
\hline $\mathrm{C}-\mathrm{C}$ & Tersoff [9] \& ZBL [10] \\
\hline Si-Si & Stillinger and Weber [11] \\
\hline Si-F, F-F & Modified SW [12,13] \\
\hline Si-B, Si-Ar, ... & ZBL \\
\hline
\end{tabular}

\section{Figure captions}

Fig. 1: Snapshots of $\mathrm{C}_{1}$ and $\mathrm{C}_{60}$ impacts on a diamond surface. Black dots represent the trajectories of projectile carbon atoms. Gray dots are displacements. For $\mathrm{C}_{1}$, the results of 60 trials are overlaid.

Fig. 2: Energy dependence of the penetration depth $R_{\mathrm{p}}$ for $\mathrm{C}_{1}$ and $\mathrm{C}_{60}$ into diamond.

Fig. 3: Profiles of shadow cones formed by carbon atoms impacting on a diamond substrate with various incident energies. The black circles indicate the position of target carbon atoms in the diamond structure.

Fig. 4: Depth profiles of displaced carbon atoms in diamond by $\mathrm{C}_{60}$ and $\mathrm{C}_{1}$ impacting with $200 \mathrm{eV} /$ atom (left) and $2 \mathrm{keV/atom} \mathrm{(right),} \mathrm{0.1ps} \mathrm{after} \mathrm{impact.}$

Fig. 5: Energy dependence of $R_{\mathrm{p}}$ enhancement factors for boron clusters.

Fig. 6: Depth profiles of vacancies (left) and interstitials (right) by boron monomer and cluster implantation.

Fig. 7: Radial distribution of substrate $\mathrm{Si}$ atoms around vacancies (left) and interstitials (right).

Fig. 8: Snapshots of $20 \mathrm{keV} \mathrm{Ar} 2000$ clusters impacting a $\mathrm{Si}(100)$ target.

Fig. 9: Snapshots and surface contours of a one-dimensional, sinusoidal silicon surface, before and after impact by $20 \mathrm{keV} \mathrm{Ar} 2000$ clusters.

Fig. 10: Surface profiles of snapshots shown in Figs. 8 and 9.

Fig. 11: Energy dependence of surface profiles (left) and crater dimensions (right) formed from Ar 2000 cluster impacts.

Fig. 12: Snapshots of various sizes of argon clusters impacting on silicon targets. Each cluster has the same total acceleration energy of $20 \mathrm{ckeV}$.

Fig. 13: Surface profiles (left) and crater depth (right) formed from clusters with various sizes accelerated to $20 \mathrm{keV}$ in total energy.

Fig. 14: Incident angle dependence of surface profiles (left) and crater depth (right) formed with $20 \mathrm{keV} \mathrm{Ar} 2000$ cluster impact.

Fig. 15: Snapshots of Ar2000 clusters impacting on Si(100) targets. The basic density of the incident cluster corresponds to the solid state of Ar (shown as (a), same as Fig. 8), and was varied by scaling the $x y$ axes only (b), $z$-axis only (c), and all xyz-axes (d).

Fig 16: Crater depth with a $20 \mathrm{keV}$ Ar2000 cluster impacting on a $\mathrm{Si}(100)$ target with various density scales $\mathrm{xy}$ and $\mathrm{z}$ that are extensions in the horizontal and vertical directions, respectively.

Fig. 17: Snapshots of neon (upper) and fluorine (lower) clusters impacting on silicon substrates.

Fig. 18: Distribution of sputtered species by neon and fluorine clusters from silicon. The values are averages after 100 impact trials for each condition.

Fig. 19: Snapshots of a $\left(\mathrm{F}_{2}\right)_{500,000}$ cluster with 1 eV/atom impacting on a $\mathrm{Si}(100)$ target. Only fluorine atoms and silicon atoms belonging to surface planes and displacement are drawn. A quarter of the target is removed.

Fig. 20: Species, energy, and size of clusters discussed in this article. 

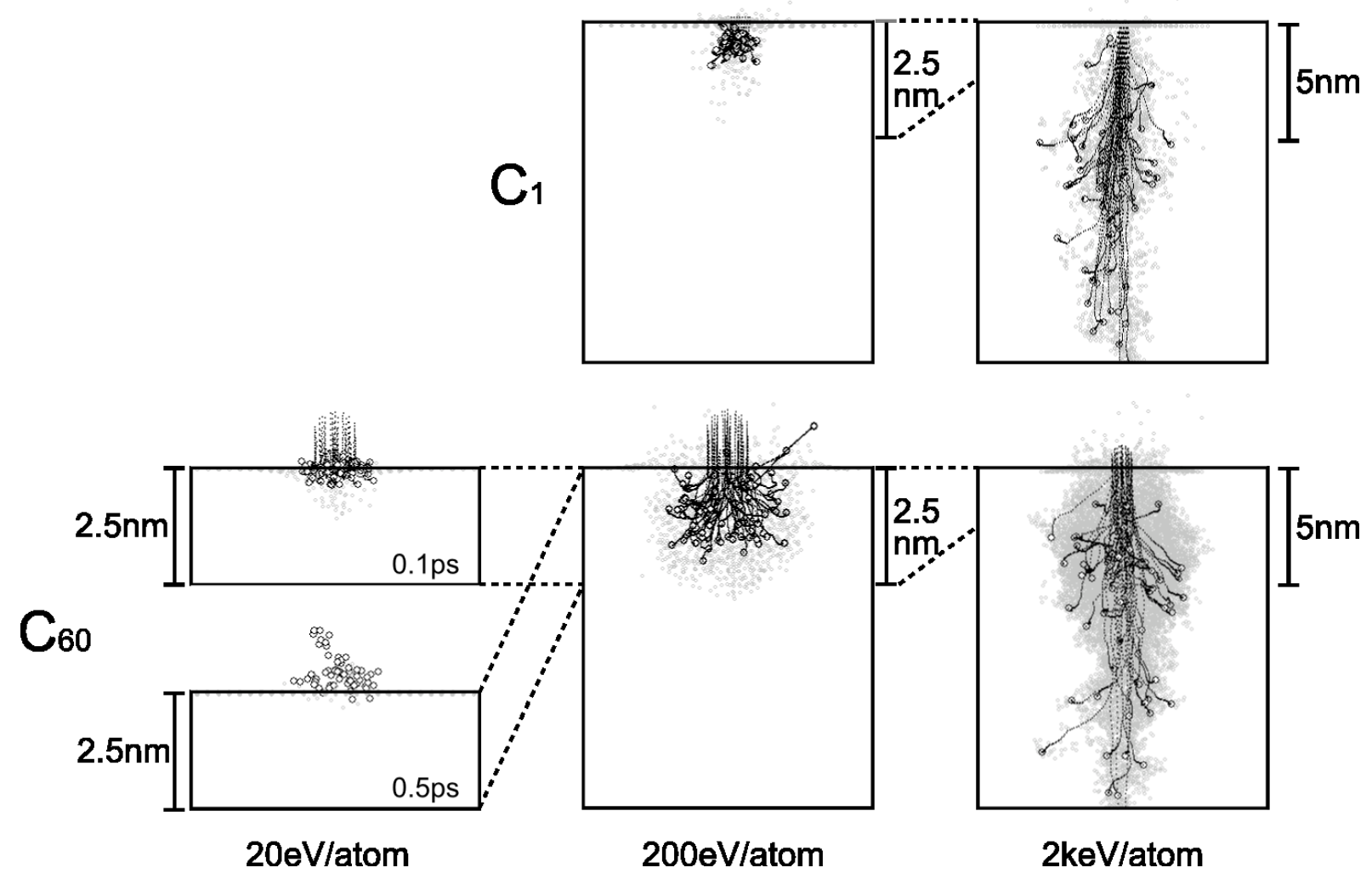

Fig. 1: Snapshots of $\mathrm{C}_{1}$ and $\mathrm{C}_{60}$ impacts on a diamond surface. Black dots represent the trajectories of projectile carbon atoms. Gray dots are displacements. For $C_{1}$, the results of 60 trials are overlaid. 


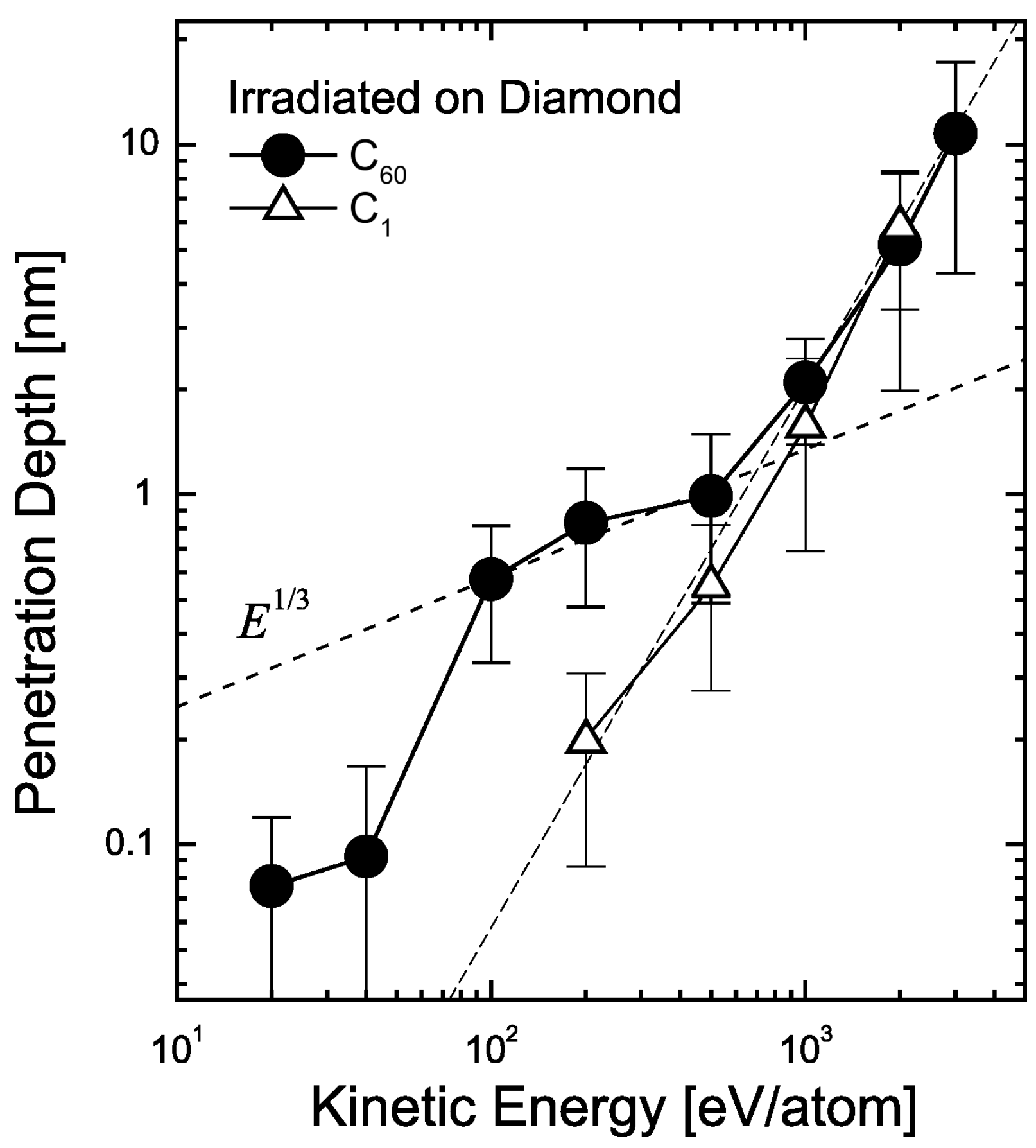

Fig. 2: Energy dependence of the penetration depth $R_{\mathrm{p}}$ for $\mathrm{C}_{1}$ and $\mathrm{C}_{60}$ into diamond. 


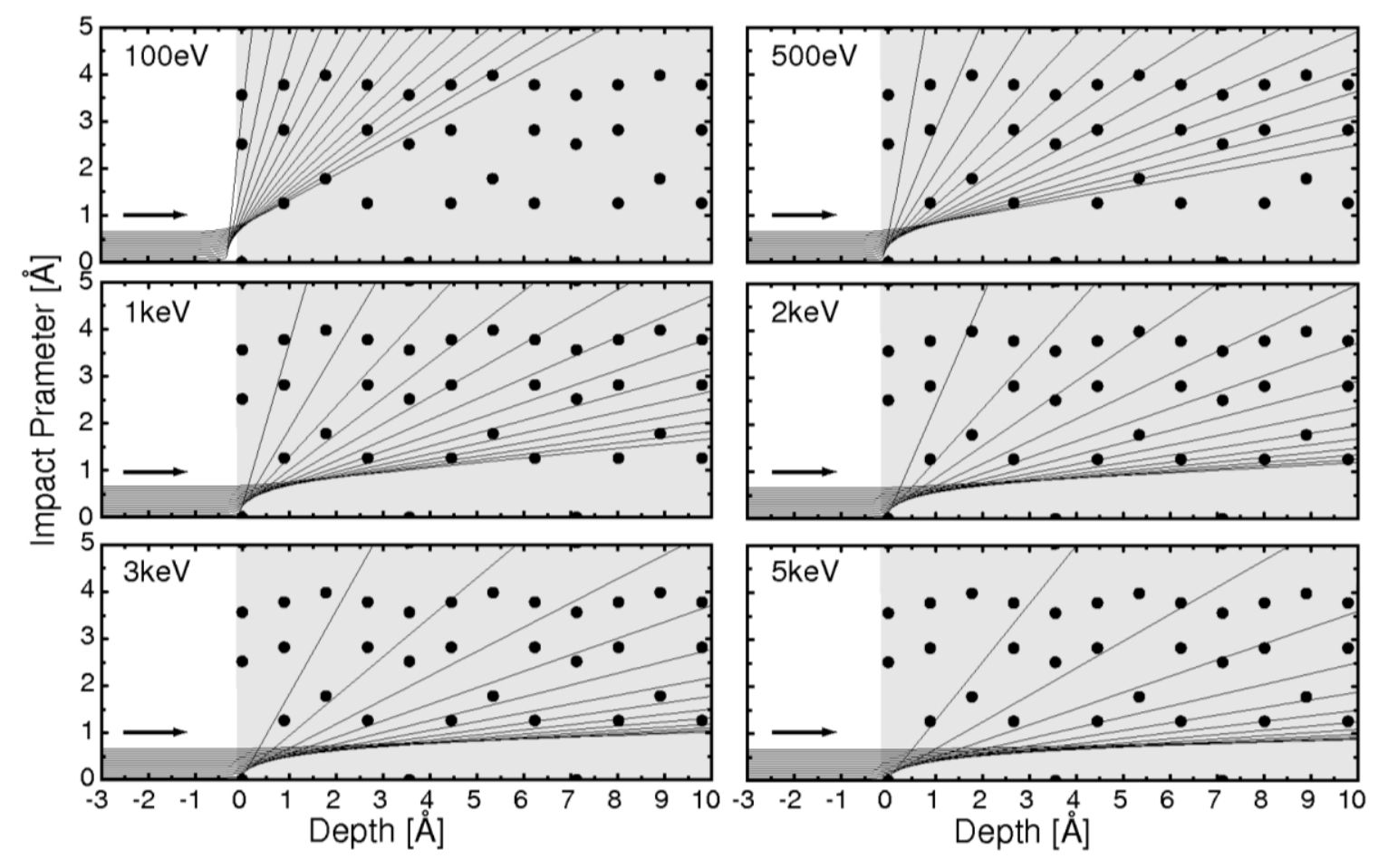

Fig. 3: Profiles of shadow cones formed by carbon atoms impacting on a diamond substrate with various incident energies. The black circles indicate the position of target carbon atoms in the diamond structure. 

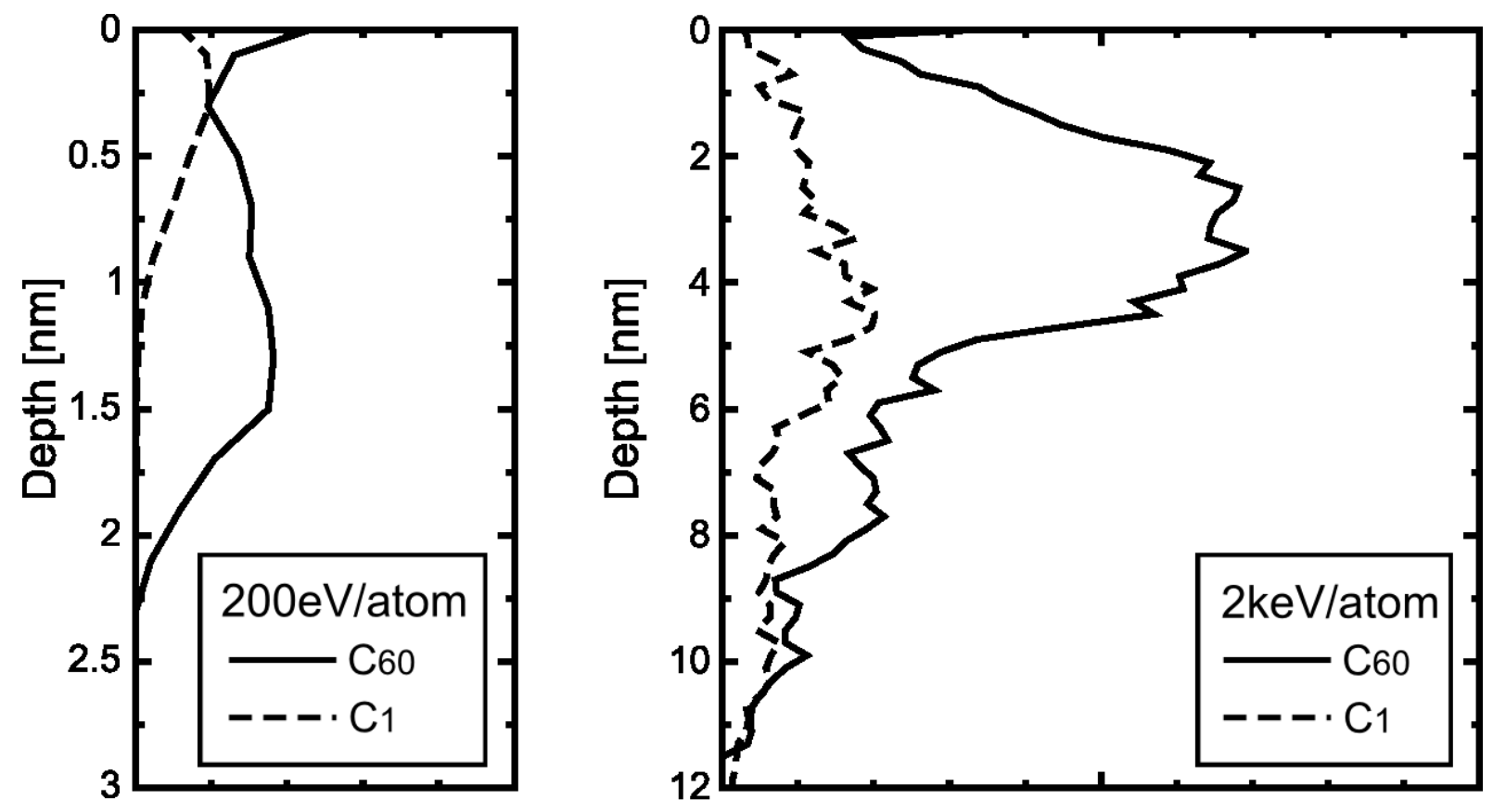

Number of Displacements [/incident atom]

Fig. 4: Depth profiles of displaced carbon atoms in diamond by $C_{60}$ and $C_{1}$ impacting with 200 $\mathrm{eV} /$ atom (left) and $2 \mathrm{keV} /$ atom (right), $0.1 \mathrm{ps}$ after impact. 


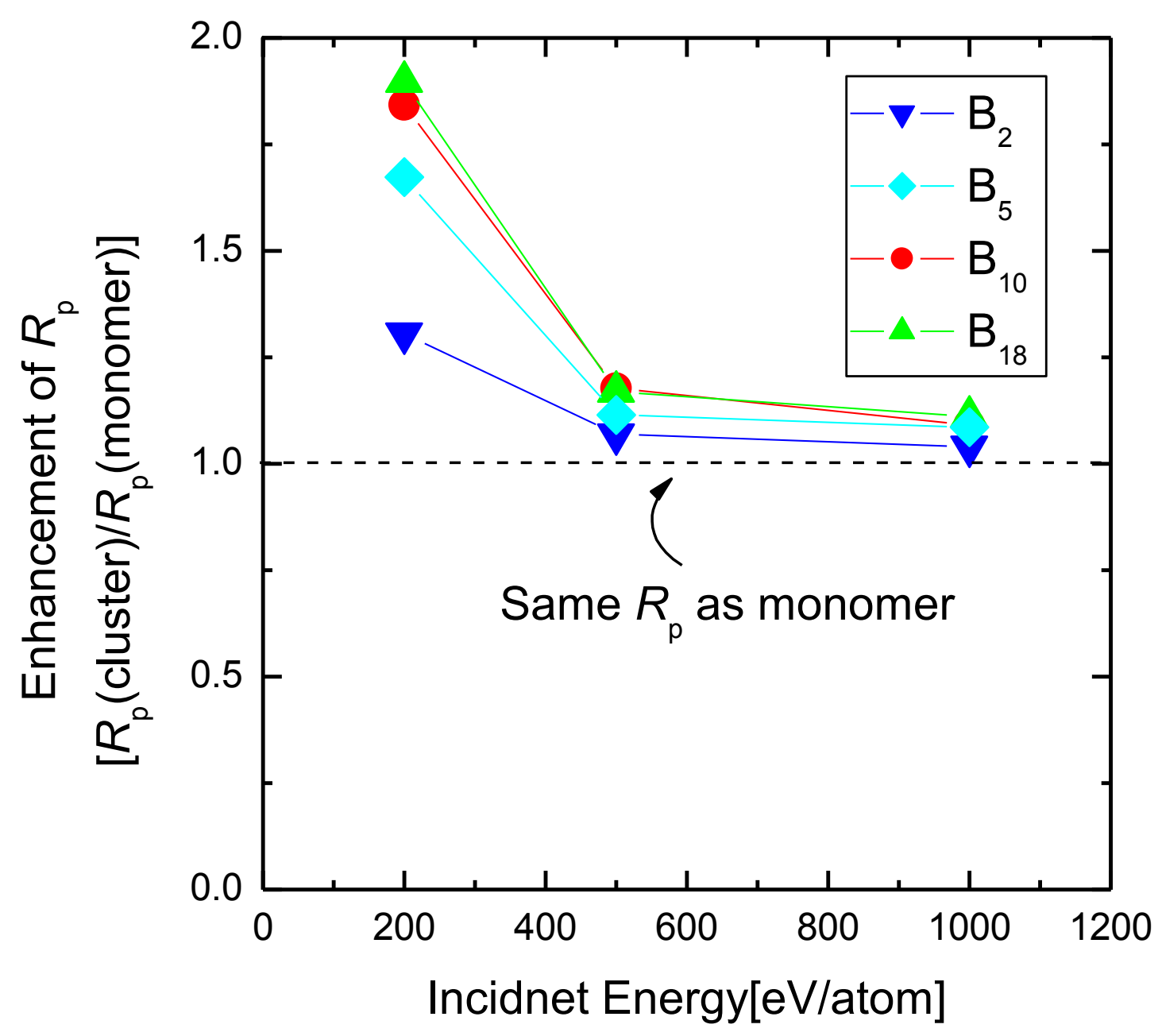

Fig. 5: Energy dependence of $R_{\mathrm{p}}$ enhancement factors for boron clusters. 

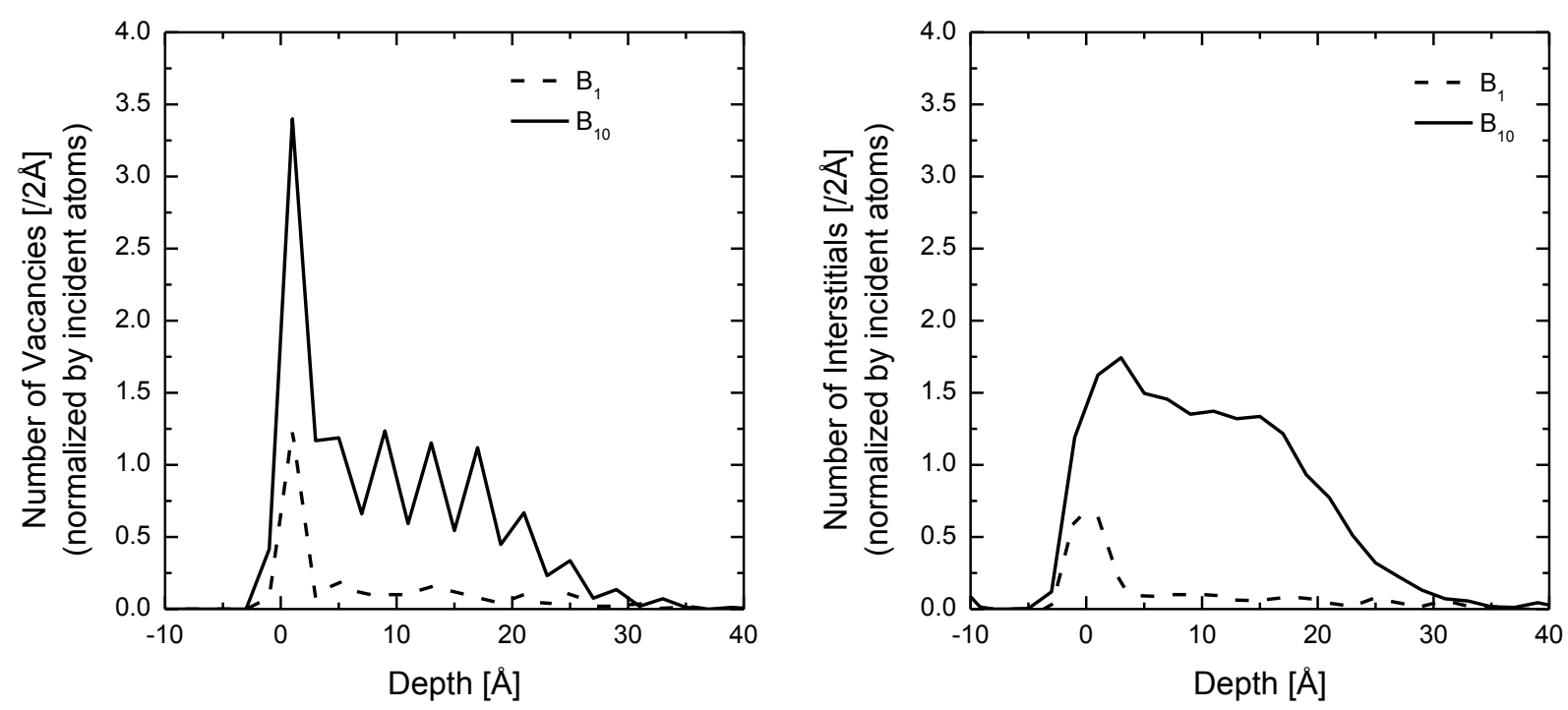

Fig. 6: Depth profiles of vacancies (left) and interstitials (right) by boron monomer and cluster implantation. 

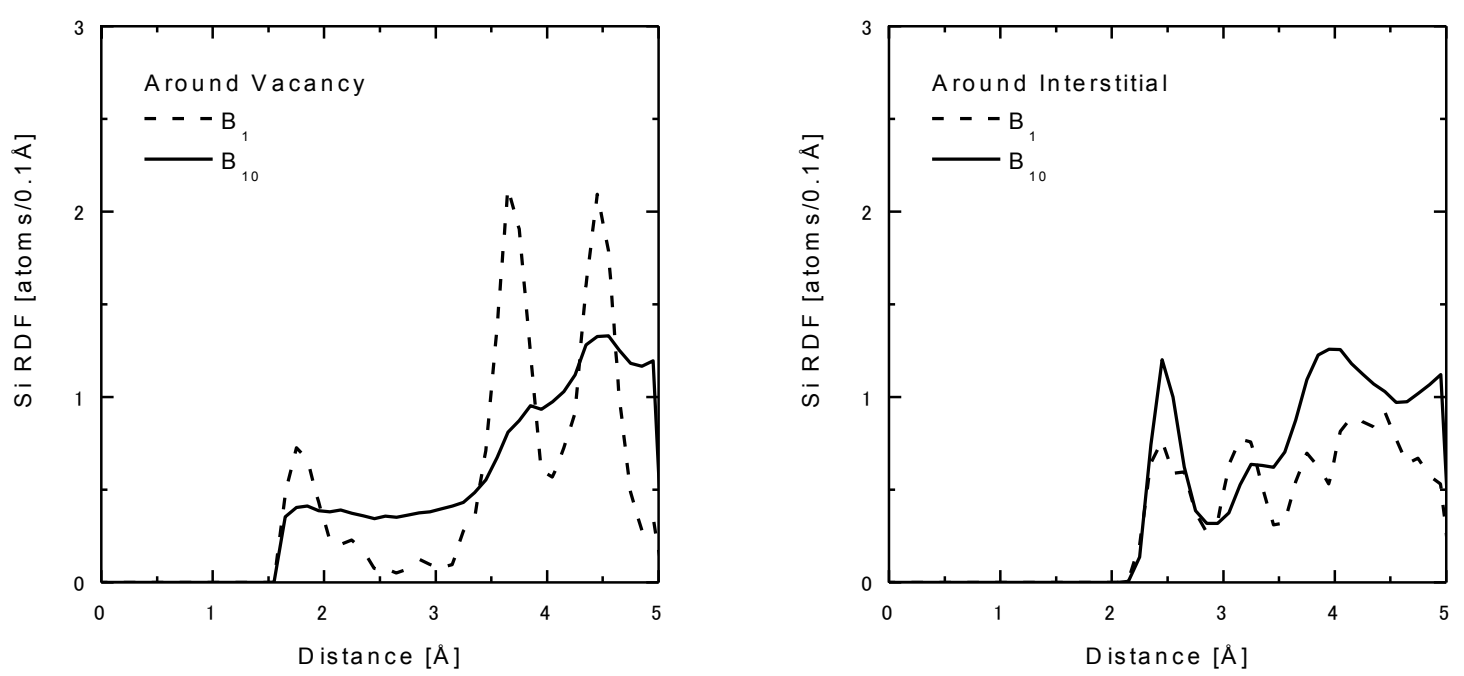

Fig. 7: Radial distribution of substrate Si atoms around vacancies (left) and interstitials (right). 

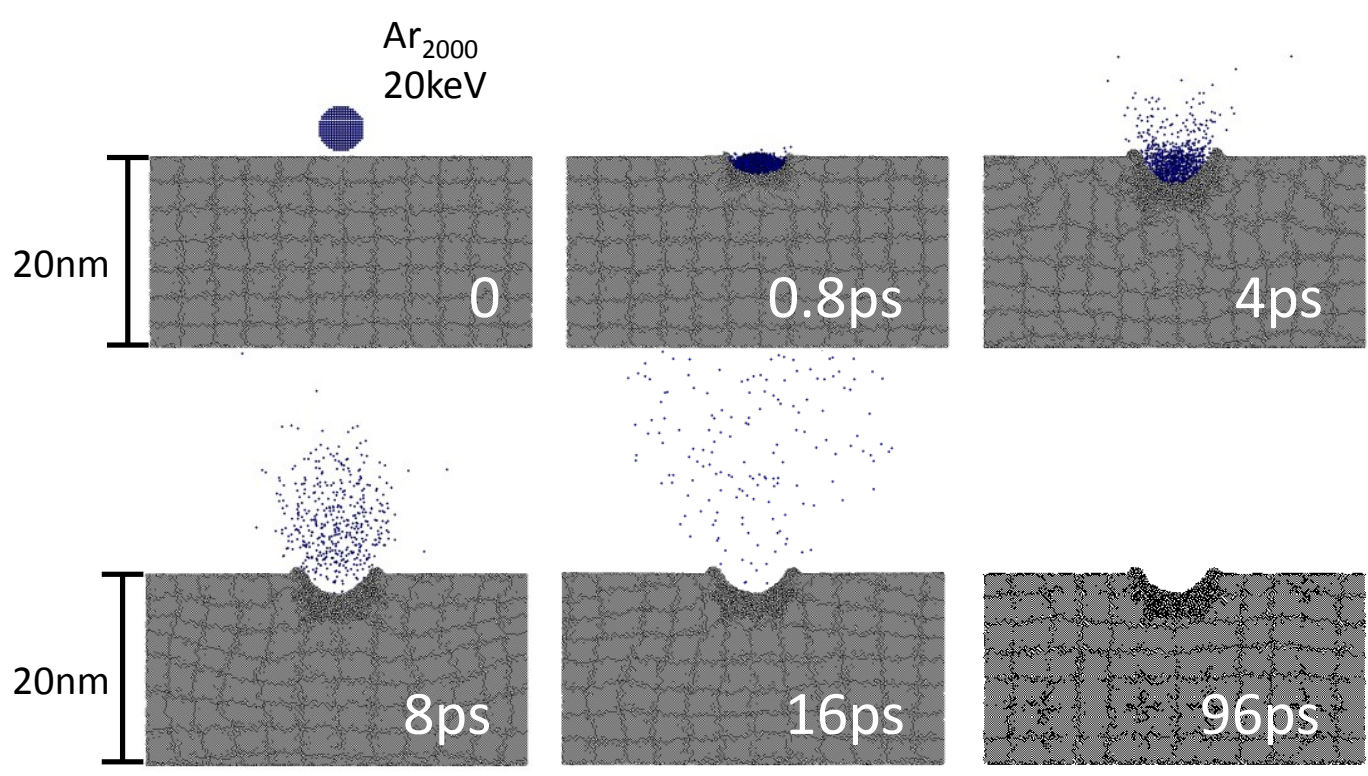

Fig. 8: Snapshots of $20 \mathrm{keV} \mathrm{Ar}_{2000}$ clusters impacting a Si(100) target. 

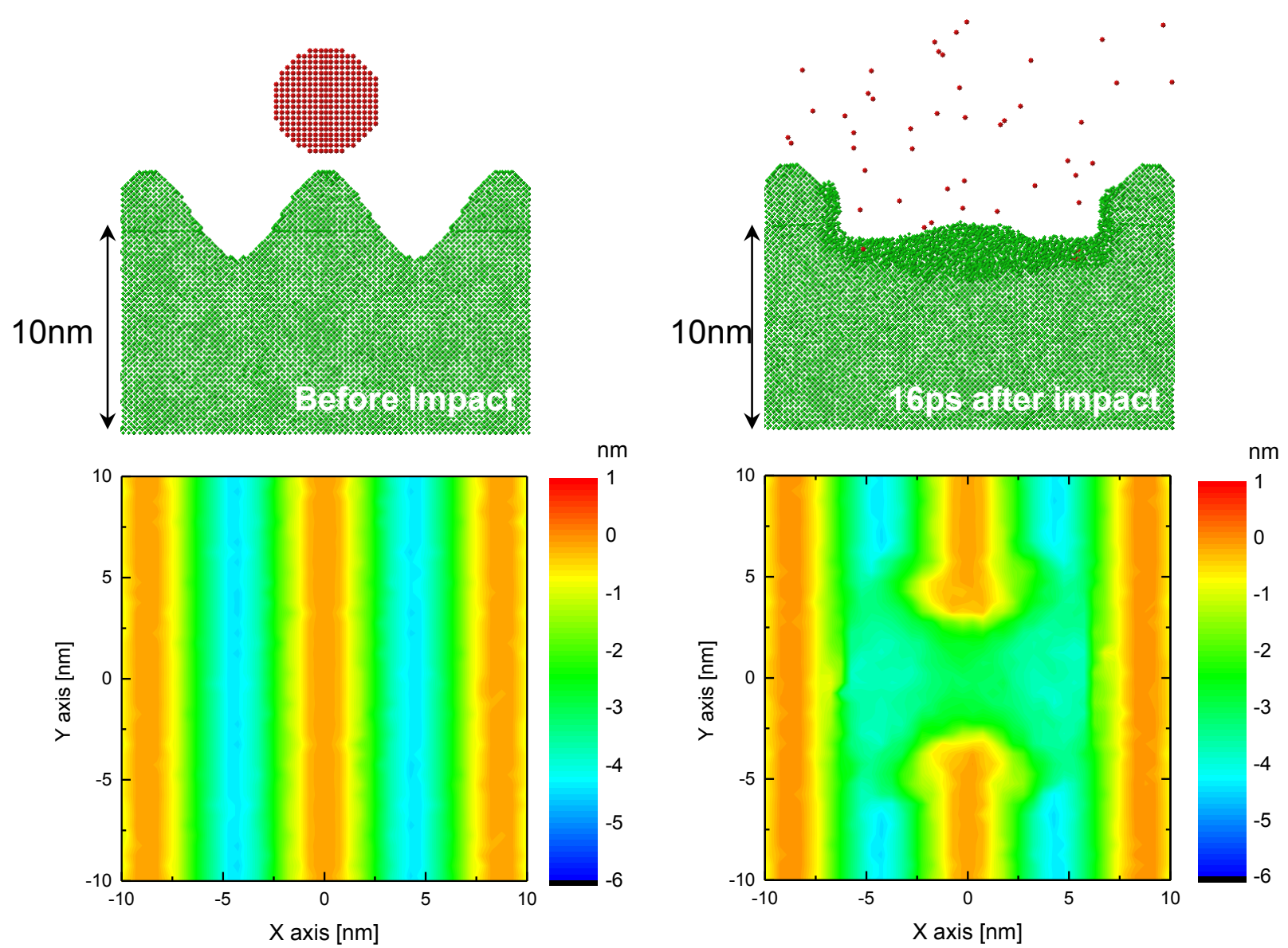

Fig. 9: Snapshots and surface contours of a one-dimensional, sinusoidal silicon surface, before and after impact by $20 \mathrm{keV} \mathrm{Ar}_{2000}$ clusters. 


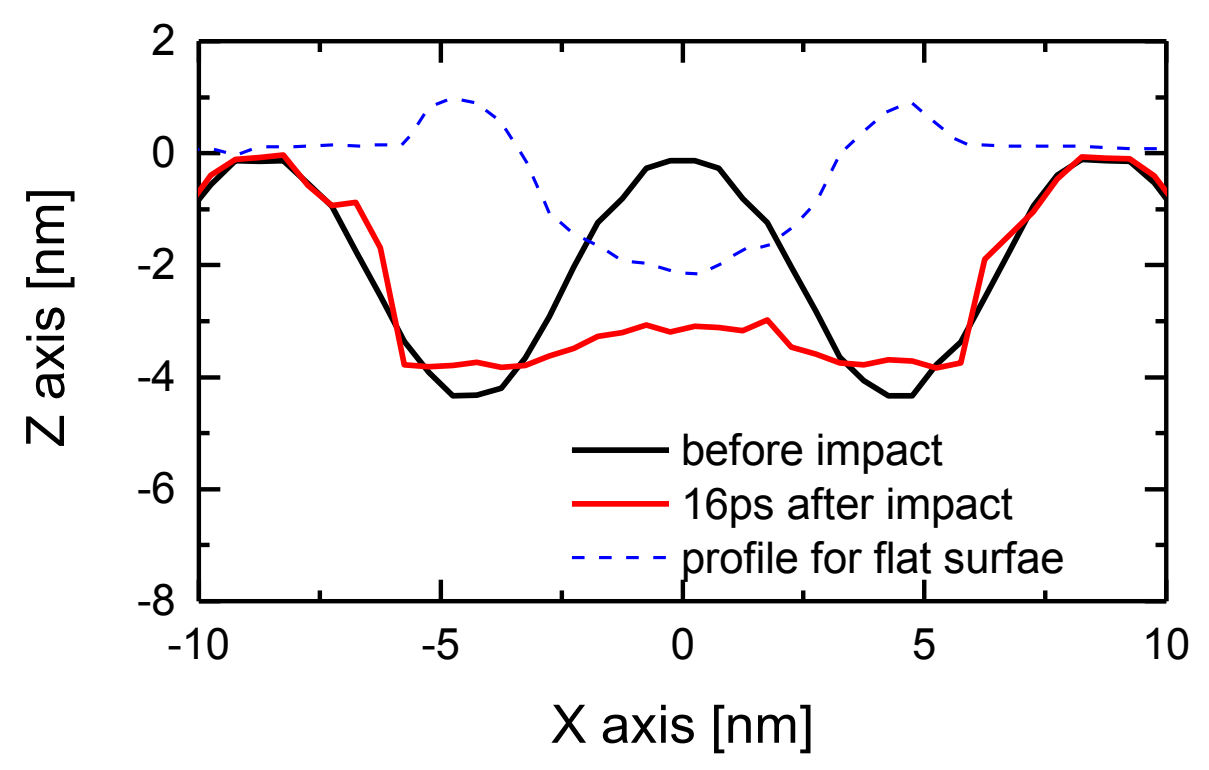

Fig. 10: Surface profiles of snapshots shown in Figs. 8 and 9. 

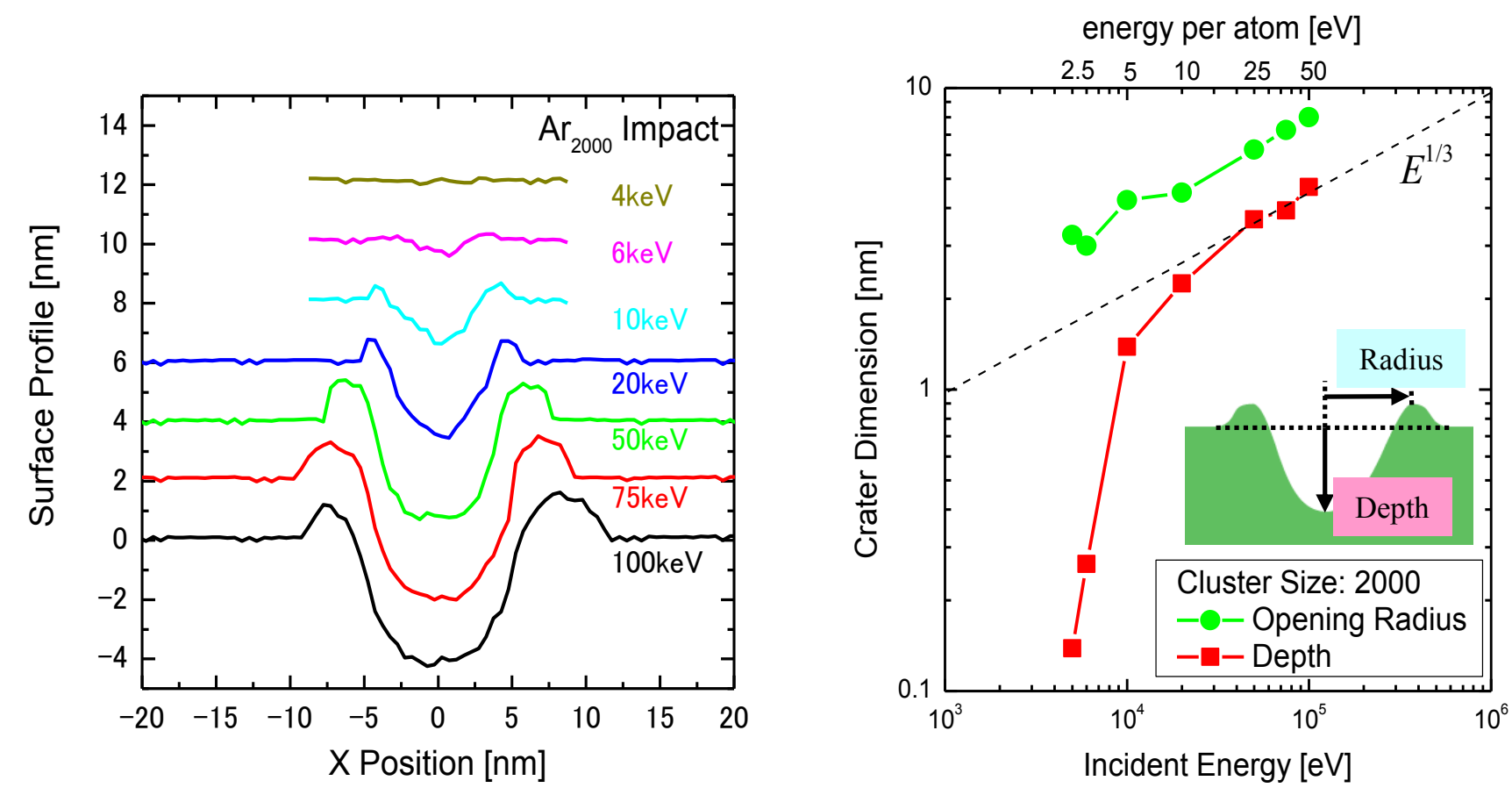

Fig. 11: Energy dependence of surface profiles (left) and crater dimensions (right) formed from $\mathrm{Ar}_{2000}$ cluster impacts. 


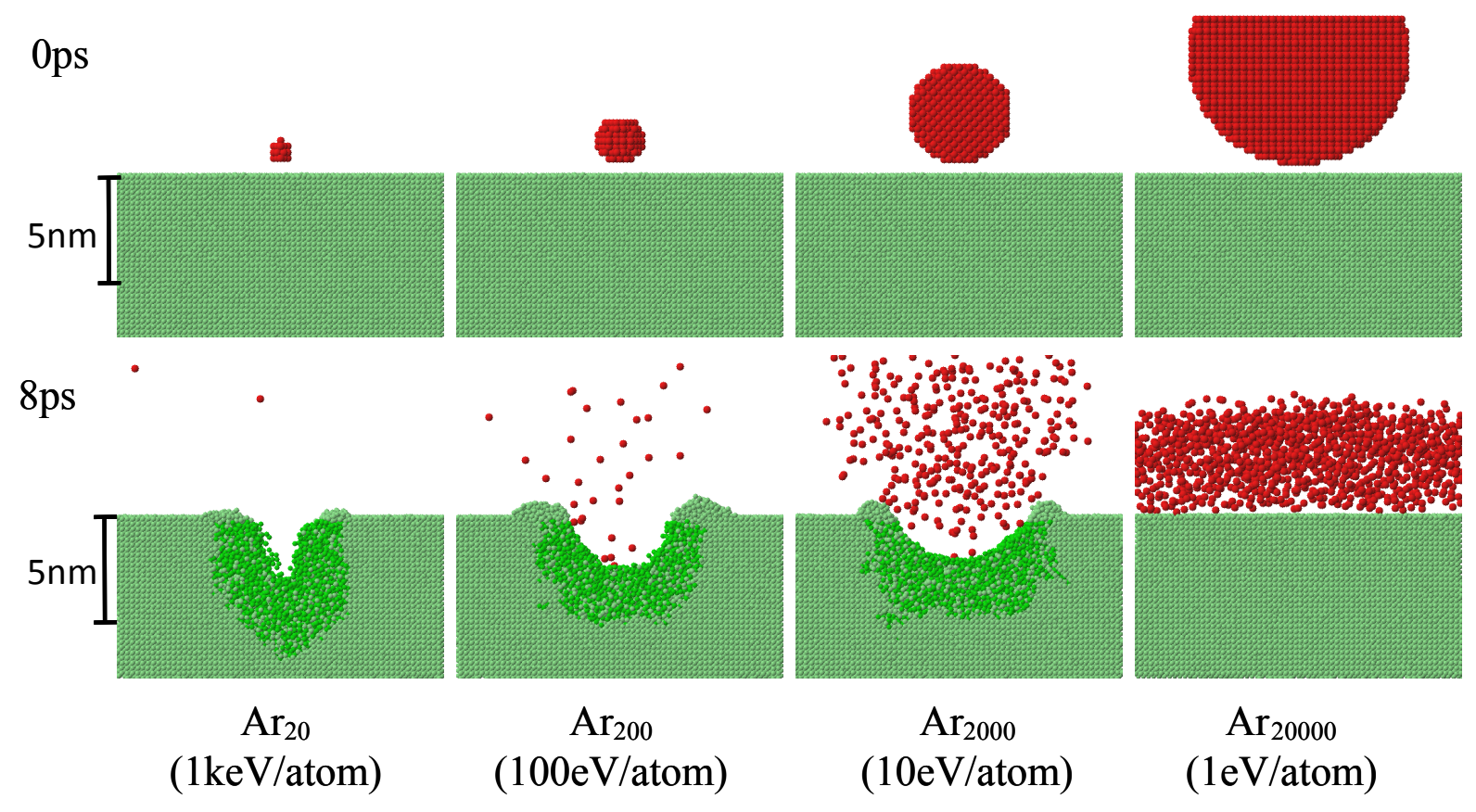

Fig. 12: Snapshots of various sizes of argon clusters impacting on silicon targets. Each cluster has the same total acceleration energy of $20 \mathrm{ckeV}$. 

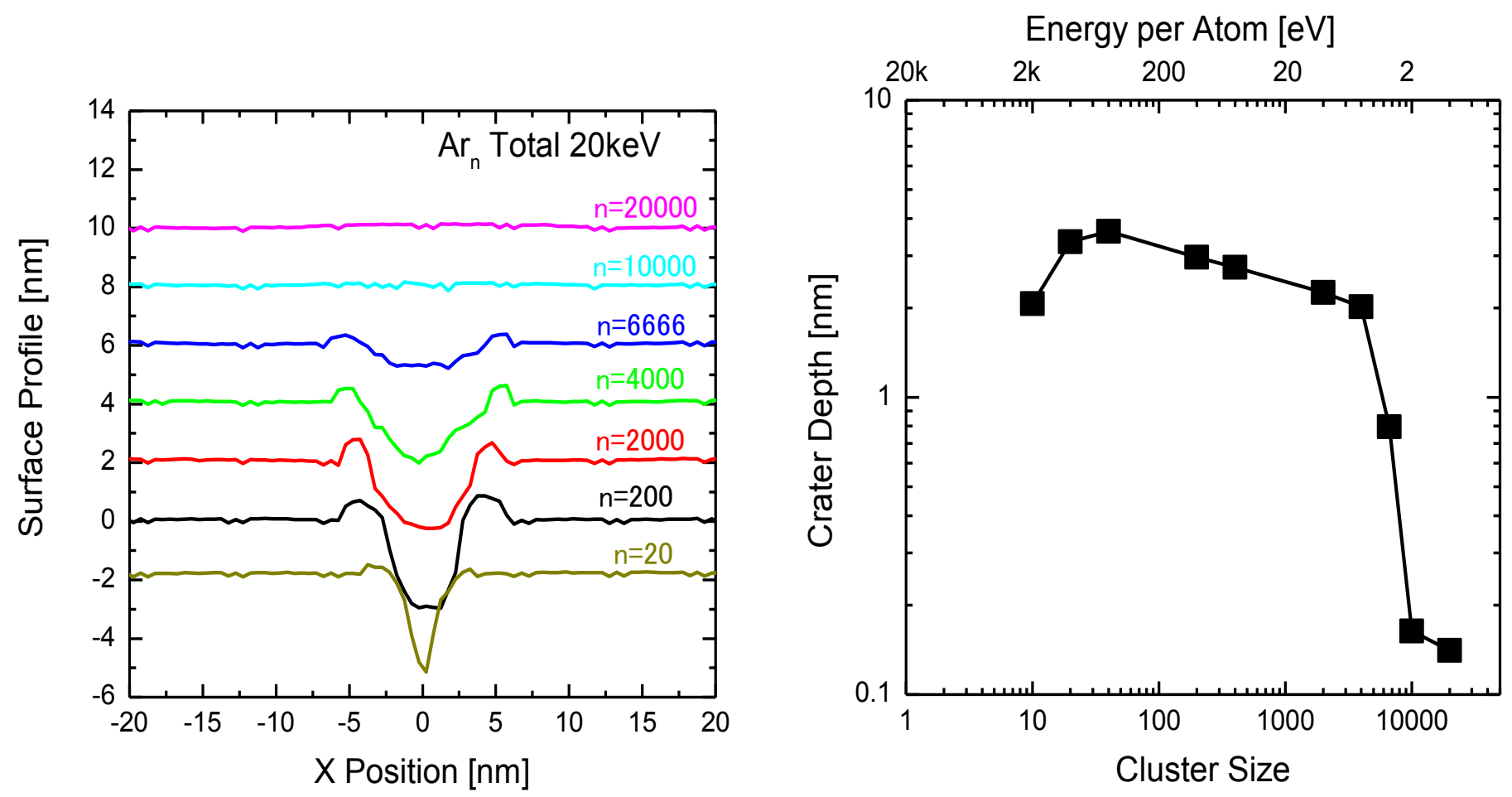

Fig. 13: Surface profiles (left) and crater depth (right) formed from clusters with various sizes accelerated to $20 \mathrm{keV}$ in total energy. 

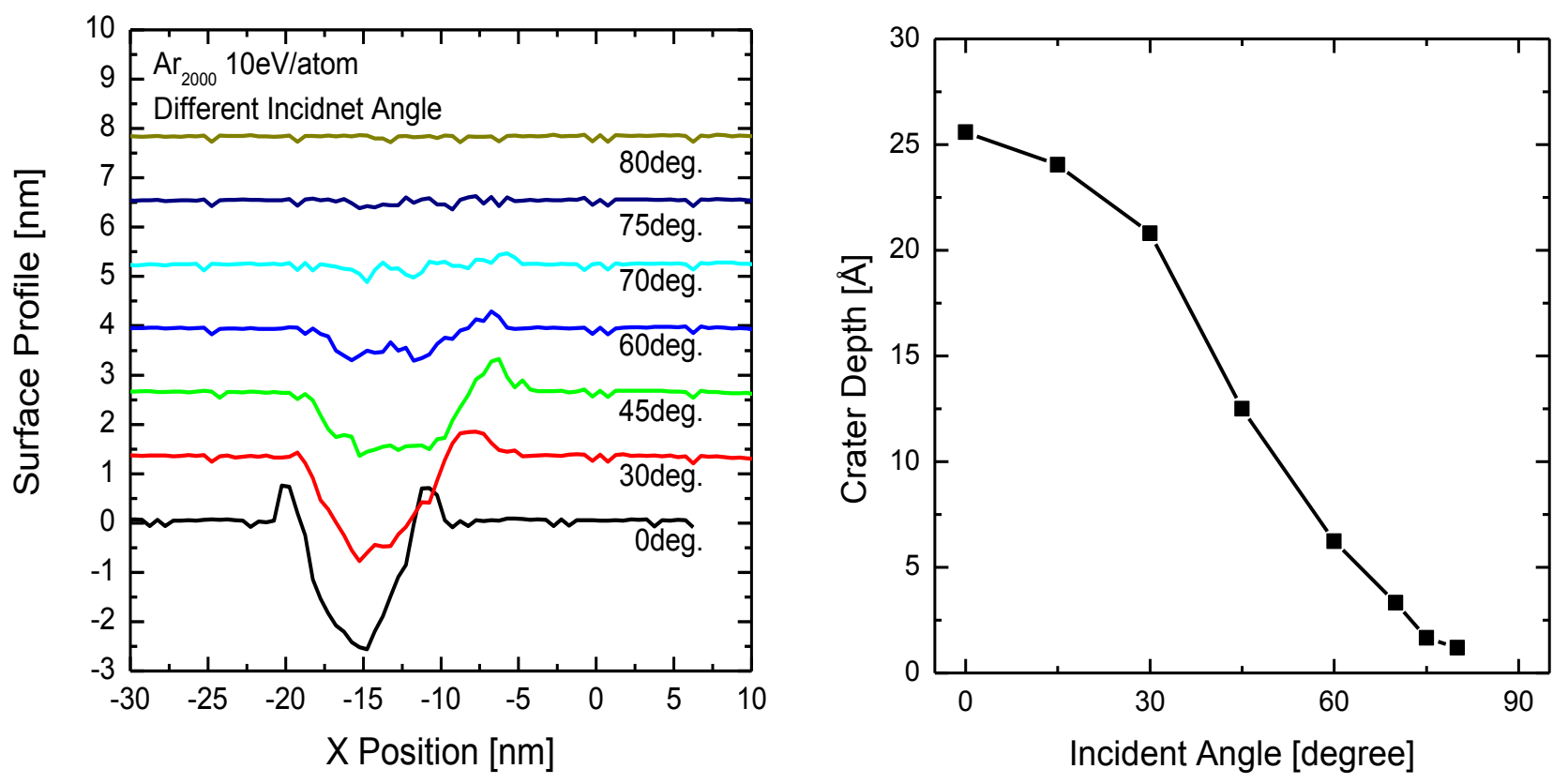

Fig. 14: Incident angle dependence of surface profiles (left) and crater depth (right) formed with $20 \mathrm{keV} \mathrm{Ar}_{2000}$ cluster impact. 
(a)

xy scale:
z scale:

$2.4 \mathrm{ps}$

$4 \mathrm{ps}$

$16 p s$
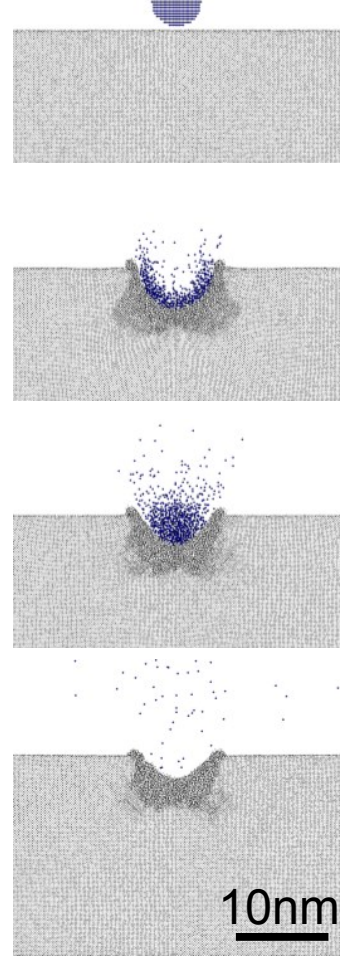

(b)

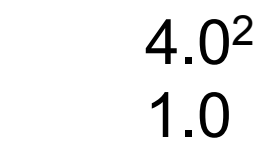

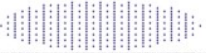
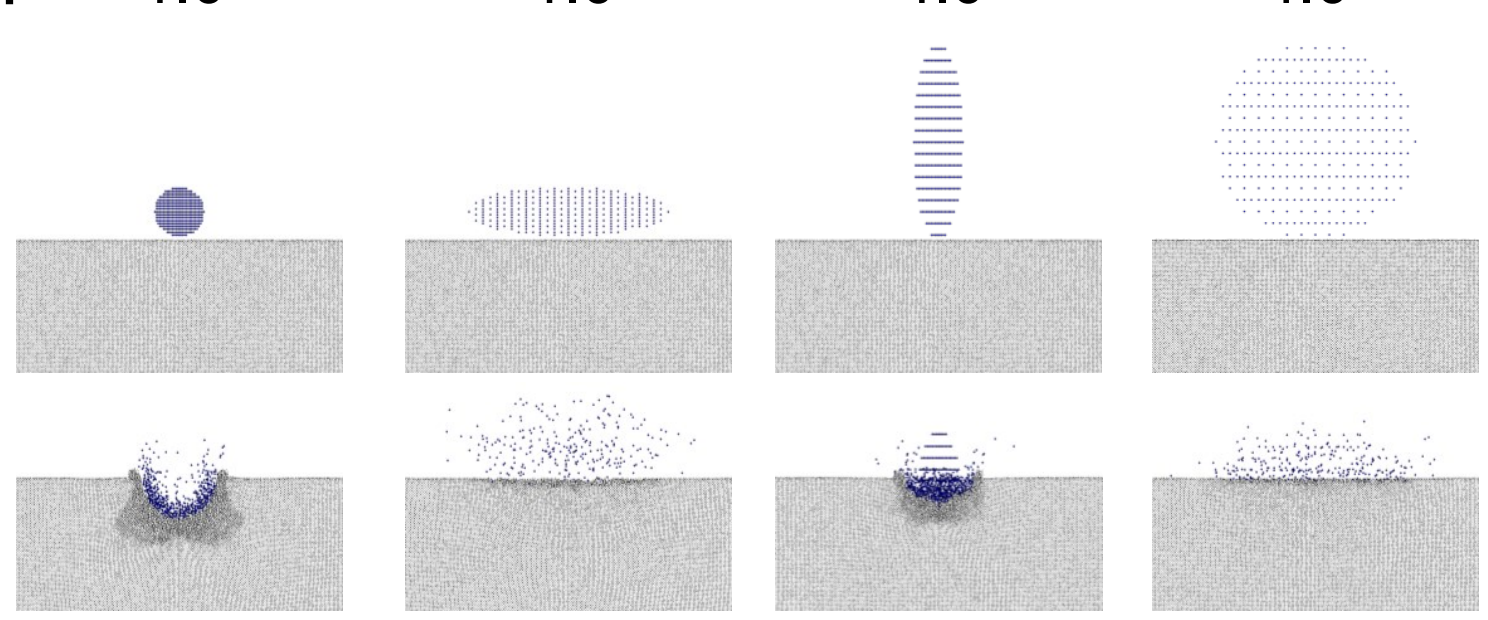

(c)

(d)

$1.0^{2}$

$4.0^{2}$

4.0
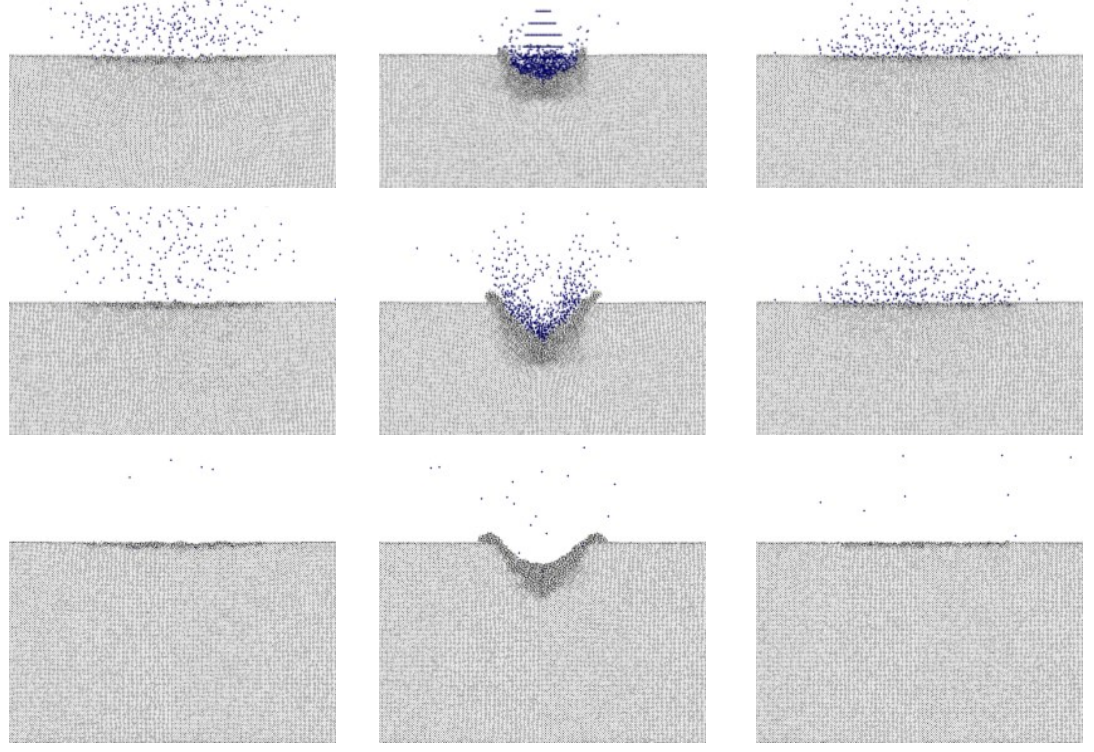

Fig. 15: Snapshots of $\mathrm{Ar}_{2000}$ clusters impacting on $\mathrm{Si}(100)$ targets. The basic density of the incident cluster corresponds to the solid state of $\operatorname{Ar}$ (shown as (a), same as Fig. 8), and was varied by scaling the $x y$-axes only (b), $z$-axis only (c), and all $x y z$-axes (d). 


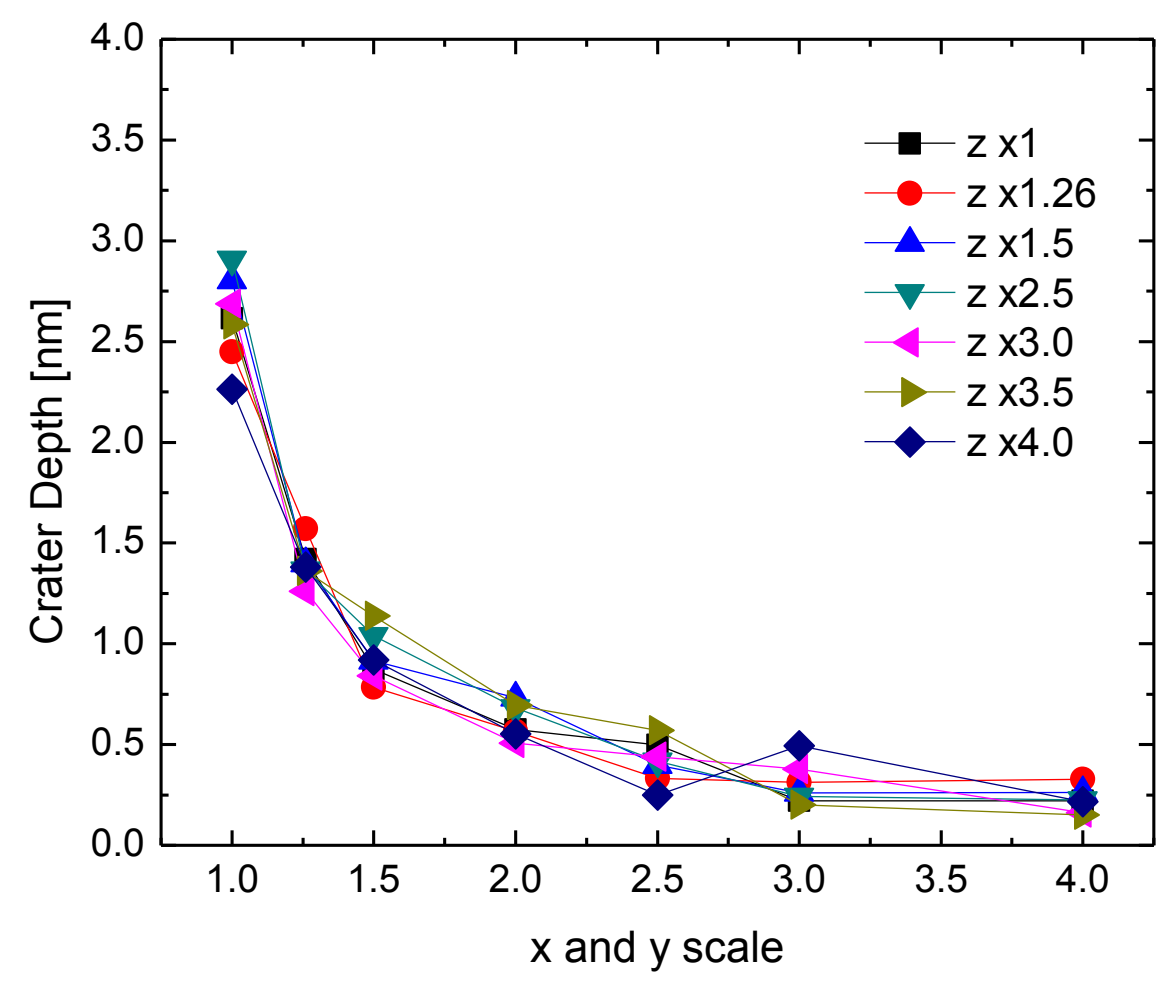

Fig 16: Crater depth with a $20 \mathrm{keV} \mathrm{Ar}_{2000}$ cluster impacting on a Si(100) target with various density scales $x y$ and $z$ that are extensions in the horizontal and vertical directions, respectively. 
Neon cluster (total $6 \mathrm{keV}, 7.7 \mathrm{ps}$ after impact)
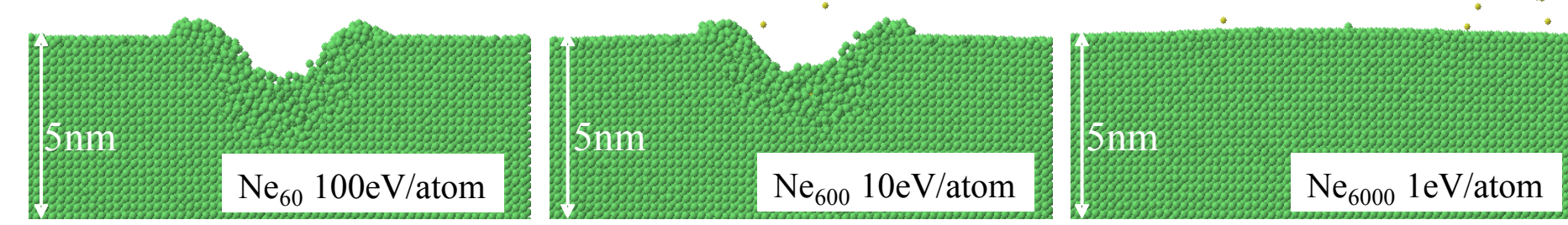

Fluorine cluster (total $6 \mathrm{keV}, 7.7 \mathrm{ps}$ after impact)
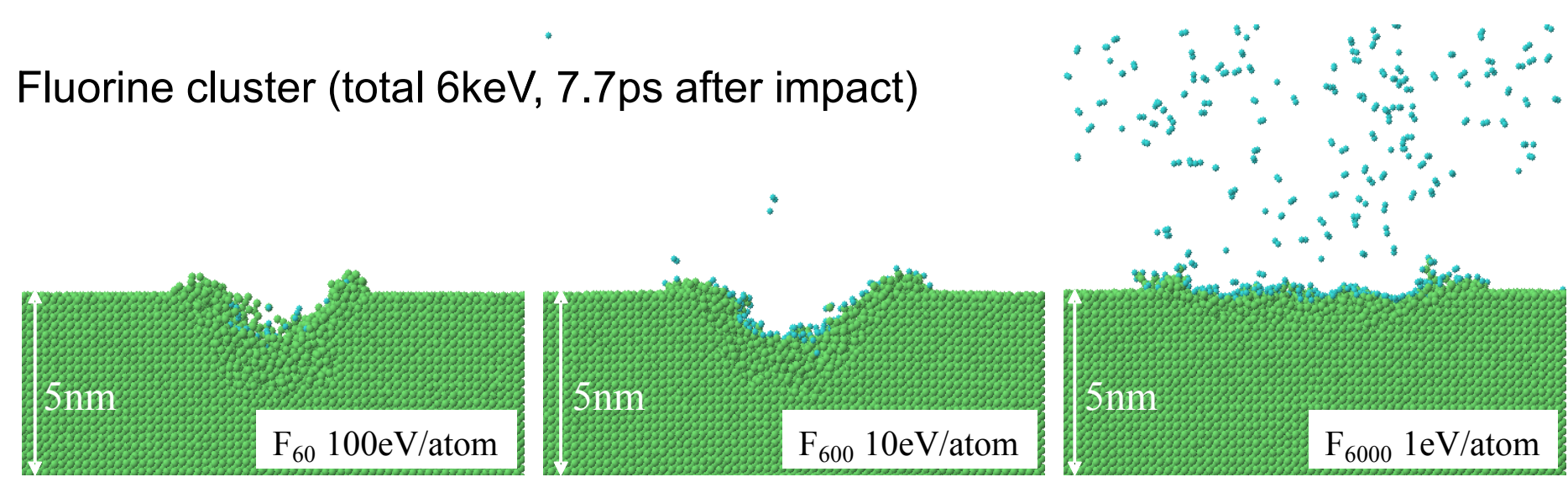

Fig. 17: Snapshots of neon (upper) and fluorine (lower) clusters impacting on silicon substrates. 


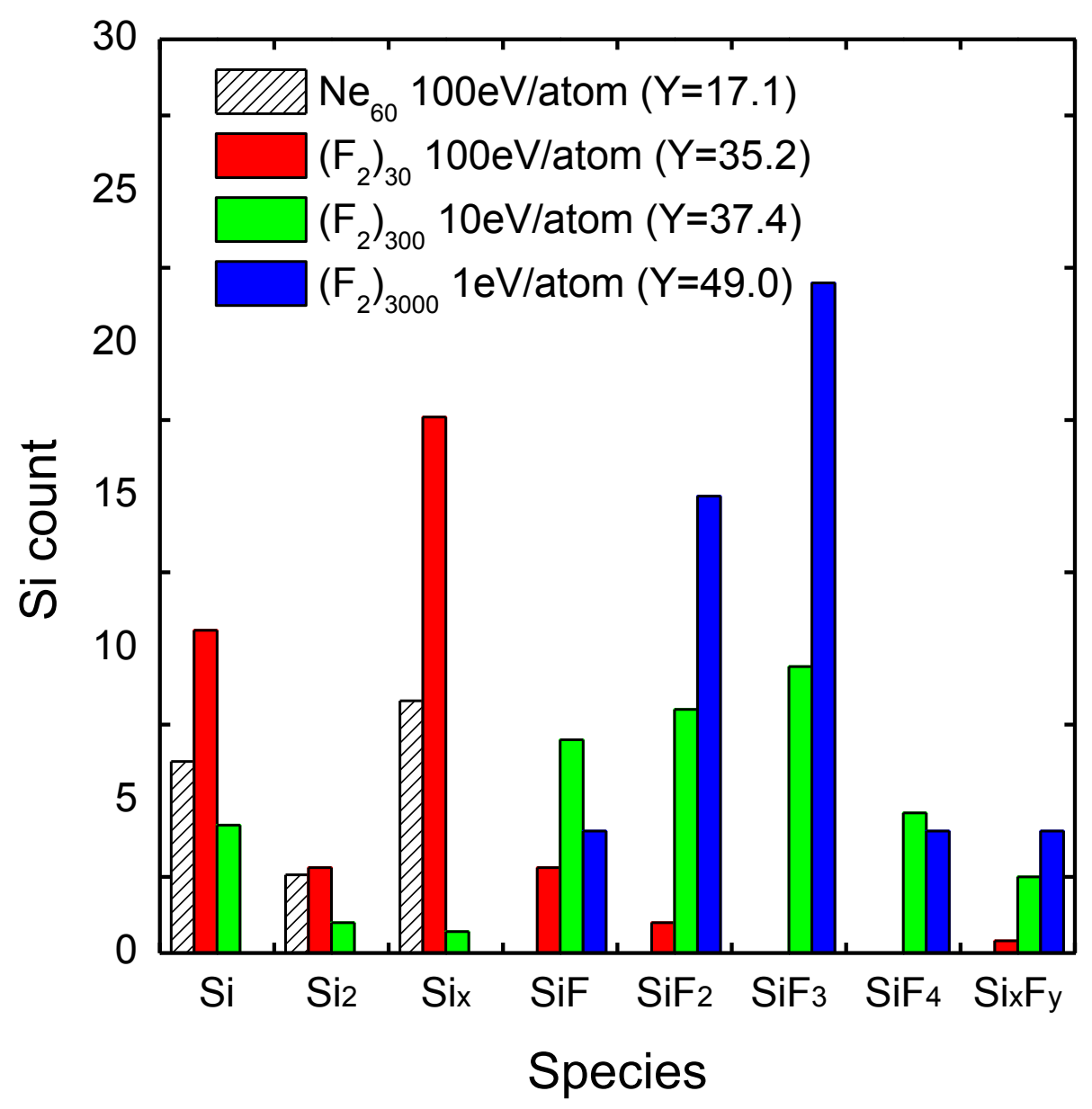

Fig. 18: Distribution of sputtered species by neon and fluorine clusters from silicon. The values are averages after 100 impact trials for each condition. 

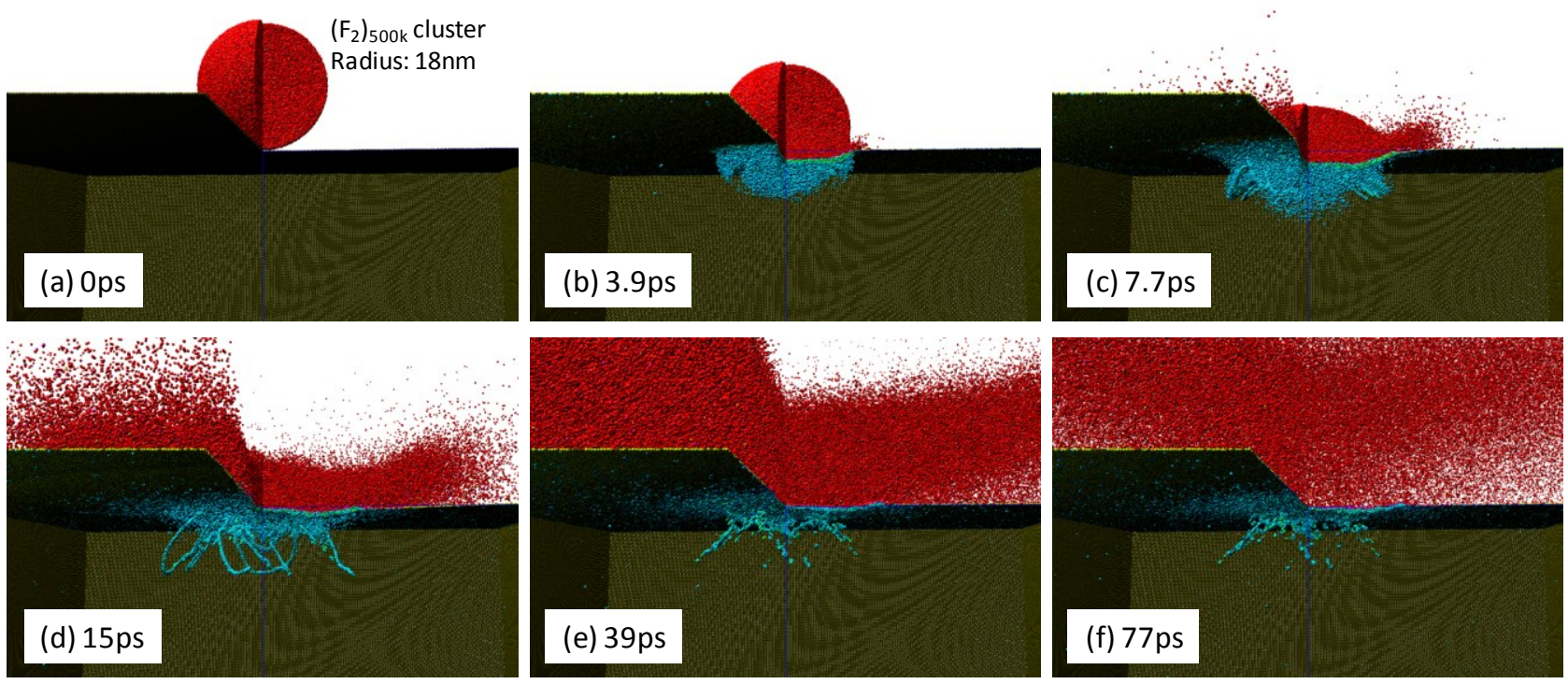

Fig. 19: Snapshots of a $\left(F_{2}\right)_{500,000}$ cluster with 1 eV/atom impacting on a Si(100) target. Only fluorine atoms and silicon atoms belonging to surface planes and displacement are drawn. A quarter of the target is removed. 


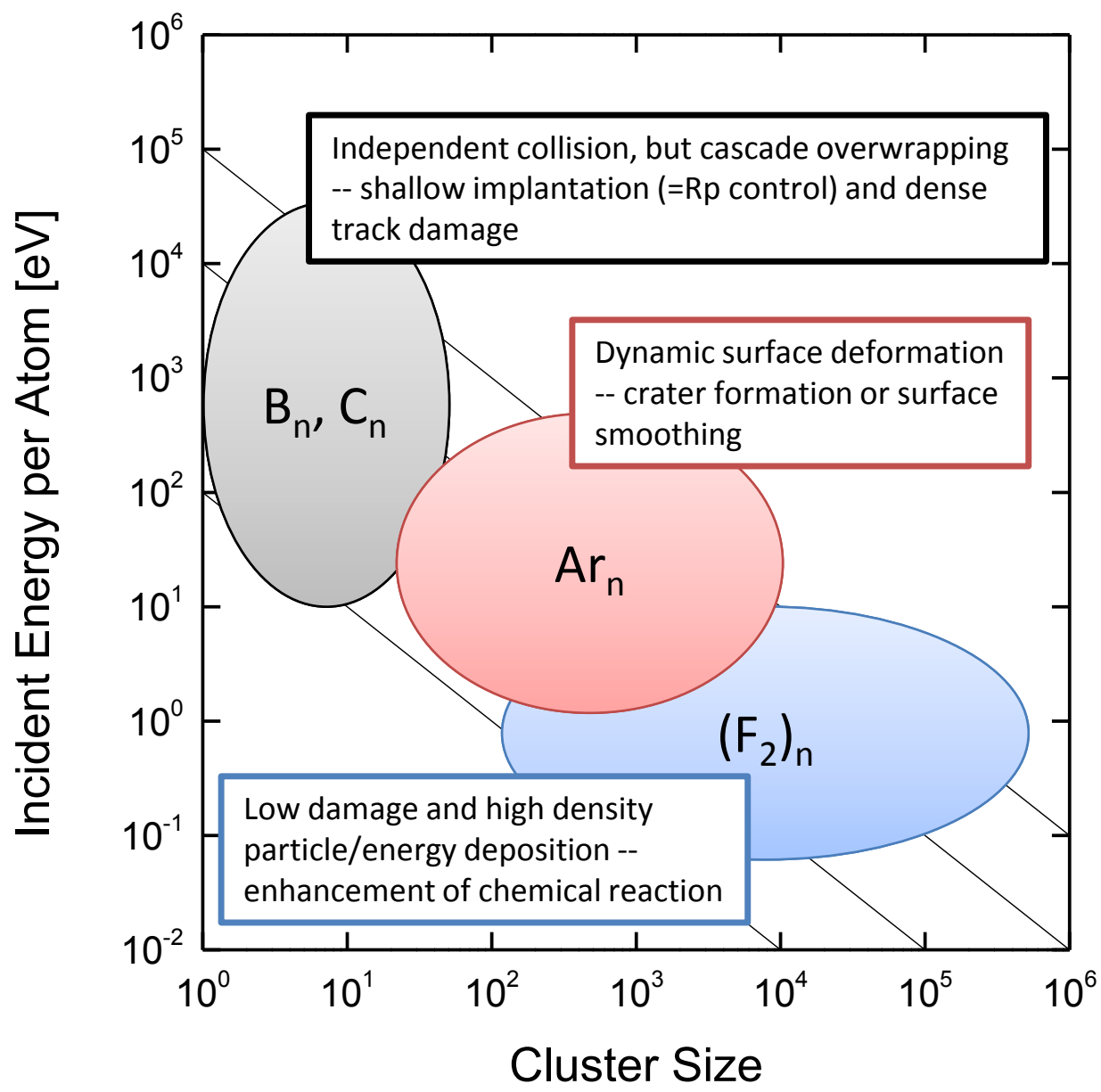

Fig. 20: Species, energy, and size of clusters discussed in this article. 